\title{
Alpha Power Poisson-G Distribution With an Application to Bur XII Distribution Lifetime Data
}

\author{
Jemilohun Vincent Gbenga ${ }^{1}$, Ipinyomi Reuben Adeyemi $^{1}$ \\ ${ }^{1}$ Department of Statistics, Afe Babalola University Ado-Ekiti, Department of Statistics, University of Ilorin, Kwara \\ State, Nigeria \\ Correspondence: Jemilohun Vincent Gbenga, Afe Babalola University Ado-Ekiti, Ekiti State, Nigeria
}

Received: December 20, 2021 Accepted: January 28, 2022 Online Published: February 13, 2022

doi:10.5539/ijsp.v11n2p8

URL: https://doi.org/10.5539/ijsp.v11n2p8

\begin{abstract}
We propose a new method of adding two shape parameters to a family of distributions for more flexibility and wider scope of applications called Alpha power Poisson-g distribution. A special case has been considered in details namely; one parameter exponential distribution. Various properties of the proposed distribution, including explicit expressions for the moments, quantiles, moment generating function, mean and median deviation, Bonferroni and Lorenz curve, order statistics and expression of the Renyi entropies are derived. The maximum likelihood estimators of unknown parameters cannot be obtained in explicit forms, and they have to be obtained by solving non-linear equations only. Further we consider an extension of the two-parameter Bur XII distribution also, mainly for data analysis purposes. Three data sets have been analyzed to show how the proposed models work in practice. We also carried out Monte Carlo simulation to further investigate the properties of the proposed method of estimation.
\end{abstract}

Keywords: Bur XII distribution, moments, Bonferroni and Lorenz curve, and maximum likelihood estimation. 8

\section{Introduction}

Several methods have been proposed and developed to generate a new generalized family of distributions. This is to address the monotone failure rate exhibited by classical distributions which often makes it not suitable to model real lie data. Generated family of continuous distributions is a new improvement for creating and extending the usual classical distributions. The newly generated families have been broadly studied in several areas as well as yield more flexibility in applications. Eugene et al. (2002) proposed the beta generated method that uses the beta distribution with parameters $\alpha$ and $\beta$ as the generator to develop the beta generated distributions. The CDF of a beta-generated random variable $X$ is defined as

$$
G(x)=\int_{0}^{F(x)} q(t) d t
$$

where $q(t)$ is the PDF of a beta random variable and $F(x)$ is the CDF of any random variable $X$. Alzaatreh et al. (2013) introduced a new method for generating families of continuous distributions called $T-X$ family by replacing the beta PDF with a PDF, $r(t)$, of a continuous random variable and applying a function $W(F(x))$ that satisfies some specific conditions.

Zografos and Balakrishnan (2009) suggested a generated family using gamma distribution which is defined as follows

$$
G_{1}(x)=\frac{1}{\Gamma(v)} \int_{0}^{-\log [1-J(x ; \xi)]} t^{v-1} e^{-t} d t
$$

Kumaraswamy generalized family provided by (Cordeiro and de Castro, 2011). Ristic and Balakrishnan, 2011) proposed an alternative gamma generator for any continuous distribution $J(x)$ which is defined as

$$
G_{1}(x)=1-\frac{1}{\Gamma(v)} \int_{0}^{-\log [1-J(x ; \xi)]} t^{v-1} e^{-t} d t
$$


Where, $\Gamma(v)=G_{1}(x)=\frac{1}{\Gamma(v)} \int_{0}^{\infty} t^{v-1} e^{-t} d t$, is the gamma function.

Further, some generated families were studied by several authors, for example, the Cordeiro and de Castro (2011) developed the Kumaraswamy-G, kummer beta by Pescim et al. (2012), exponentiated generalized class by Cordeiro et al. (2013),), Weibull-G by Bourguignon et al. (2014), exponentiated half-logistic by Cordeiro et al. (2014), transmuted exponentiated generalized-G by Yousof et al. (2015), the type I half-logistic by (Cordeiro et al., 2015), the Kumaraswamy Weibull by Hassan and Elgarhy (2016), transmuted geometric-G by Afify et al. (2016a), Kumaraswamy transmuted-G family of distribution was studied by Afify et al. (2016b). Nofal et al. (2017) developed the transmuted-G family Alizadeh et al. (2017) developed the generalized odd generalized exponential, exponentiated Weibull- $\mathrm{H}$ by Cordeiro et al. (2017), alpha power transformation Mahdavi and Kundu (2017), exponentiated generalized-G Poisson by Aryal and Yousof (2017), Alizadeh et al. (2018) proposed and studied transmuted Weibull-G, Marshall-Olkin generalized-G Poisson by Korkmaz et al. (2018). Oluyede, et al. (2018) introduced the gamma Weibull-G and odd Lomax-G family by Cordeiro et al. (2019).

The aim of this paper is to introduce two extra parameters to a family of distributions functions to bring more flexibility and enhance the scope of applications to the given family. We call this new method as Alpha Power Poisson-g family of (APP-G) distribution. The proposed APP-G distribution is tractable and very easy to use; hence it can be used quite effectively to model data exhibiting different shapes of the hazard function. We have use the APPG method to a two-parameter Bur XII distribution, and generated a four-parameter Alpha power Bur XII Poisson (APBXIIP) distribution with more modeling potentials. It is observed that the four-parameter APBXIIP distribution has several desirable properties. The PDF and the hazard functions of APBXIIP distribution can take similar shapes as the Weibull, Gamma or logistic distribution. The PDF of APBXIIP distribution can be expressed in explicit form; hence it can be used quite conveniently for analyzing censored data exhibiting non-monotone failure rate.

This paper is organized as follows. In section 2, we introduced the Alpha power Poisson-g family of distribution are examined. The Alpha Power Bur XII Poisson distribution, survival hazard rate, hazard function, and mixture representation of $A P B X I I P$ distribution are given in section 3. Section 4 contains the ordinary and incomplete moments, mean and median deviation, moment generating function, Bonferroni and Lorenz curves. Renyi entropy and Order statistics are considered in section 5. Monte Carlo simulation study is conducted to examine the Absolute bias and mean square error of the maximum likelihood estimators. Also, the results on the estimation of the parameters of the $A P B X I I P$ model via the maximum likelihood estimation technique is given in section 6 which also includes real data applications. Concluding remarks is given in section 7.

\section{Alpha Power Poisson-g Method}

Suppose that a system has $\mathrm{N}$ subsystems functioning independently at a given time where $N$ has zero truncated Poisson (ZTP) distribution with parameter $\theta$. It is the conditional probability distribution of a Poisson-distributed random variable, given that the value of the random variable is not zero. The probability mass function (pmf) of $N$ is given by

$$
P(N=n)=\frac{1}{\left(1-e^{-\theta}\right)} \frac{e^{-\theta} \theta^{n}}{n !}, \quad n=1,2, \ldots
$$

the expected value and variance are respectively given by

$$
E(N)=\frac{\theta}{1-e^{-\theta}}
$$

And

$$
\operatorname{Var}(N)=\frac{\theta+\theta^{2}}{1-e^{-\theta}}-\frac{\theta^{2}}{\left(1-e^{-\theta}\right)^{2}}
$$

Suppose that the failure time of each subsystem has the Alpha power transformed-G (APT-G) distribution defined by the cumulative distribution function (CDF) and probability density function (PDF) are respectively given by

$$
H_{A P T}(x ; \alpha, \xi)=\left\{\begin{array}{rc}
\frac{\alpha^{H(x)}-1}{\alpha-1}, & \text { if } \alpha>0, \alpha \neq 1 \\
H(x), & \alpha=0
\end{array}\right.
$$


and

$$
h_{A P T}(x ; \alpha, \xi)=\left\{\begin{aligned}
\frac{\log \alpha}{\alpha-1} h(x) \alpha^{H(x)}, & \text { if } \alpha>0, \alpha \neq 1 \\
H(x), & \text { if } \alpha=0
\end{aligned}\right.
$$

where $\alpha$ is the additional shape parameter. Let $Z_{i}$ denote the failure time of the $i^{\text {th }}$ subsystem and let $X=$ $\min \left\{Z_{1}, \ldots, Z_{N}\right\}$. Then the conditional CDF of $X$ given $N$ is

$$
F(x / N)=1-P(X>x / N)=1-\left(1-H_{A P T}(x ; \alpha, \xi)\right)^{N}
$$

Therefore, the unconditional cdf of X, as described in Ramos et al. (2015), can be expressed as

$$
F(x ; \alpha, \theta, \xi)=\left[\frac{1}{1-e^{-\theta}}\right]\left[1-e^{-\theta\left(\frac{\alpha^{H(x)}-1}{\alpha-1}\right)}\right]
$$

The cdf in (9) is called the Alpha power G Poisson (APGP) family of distributions. The corresponding pdf is

$$
f(x ; \alpha, \theta, \xi)=\left[\frac{\theta \log (\alpha)}{\left(1-e^{-\theta}\right)(\alpha-1)}\right] h(x) \alpha^{\bar{H}(x)} e^{-\theta\left(\frac{\alpha^{\bar{H}(x)}-1}{\alpha-1}\right)}
$$

An expression for the survival and the hazard function for APGP family of distribution is respectively given by

$$
S(x ; \alpha, \theta, \xi)=1-\left[\frac{1}{1-e^{-\theta}}\right]\left[1-e^{-\theta\left(\frac{\alpha^{\bar{H}(x)}-1}{\alpha-1}\right)}\right]
$$

And

Using the power series expansion

$$
\bar{h}(x ; \alpha, \theta, \xi)=\frac{\theta \log (\alpha) \bar{h}(x) \alpha^{\bar{H}(x)} e^{-\theta\left(\frac{\alpha^{\bar{H}(x)}-1}{\alpha-1}\right)}}{\left(1-e^{-\theta}\right)(\alpha-1)\left\{1-\left[\frac{1}{1-e^{-\theta}}\right]\left[1-e^{-\theta\left(\frac{\alpha^{\bar{H}(x)-1}}{\alpha-1}\right)}\right]\right\}}
$$

$$
e^{x}=\sum_{v=0}^{\infty} \frac{x^{v}}{v !}
$$

we express the PDF in (12) as

$$
f(x ; \alpha, \theta, \xi)=\left[\frac{\theta \log \alpha}{\left(1-e^{-\theta}\right)}\right] \bar{h}(x) \alpha^{\bar{H}(x)} \sum_{i=0}^{\infty} \frac{(-\theta)^{i}(-1)^{i}}{i !(\alpha-1)^{i+1}}\left(1-\alpha^{\bar{H}(x)}\right)^{i}
$$

Also, applying series expansion given by

$$
(1-p)^{v}=\sum_{j=0}^{\infty}(-1)^{j}\left(\begin{array}{l}
v \\
j
\end{array}\right) p^{v}
$$

We have,

$$
f(x ; \alpha, \theta, \xi)=\left[\frac{\theta \log \alpha}{\left(1-e^{-\theta}\right)}\right] \bar{h}(x) \sum_{i, j=0}^{\infty} \frac{(-\theta)^{i}(-1)^{i+j}}{i !(\alpha-1)^{i+1}}\left(\begin{array}{l}
i \\
j
\end{array}\right) \alpha^{(j+1) \bar{H}(x)}
$$

Since, 


$$
\alpha^{u}=\sum_{t=0}^{\infty} \frac{(\log \alpha)^{t}}{t !} u^{t}
$$

Finally, we have

$$
f(x ; \alpha, \theta, \xi)=\left[\frac{\theta}{\left(1-e^{-\theta}\right)}\right] \bar{h}(x) \sum_{i, j, k=0}^{\infty} \frac{(-\theta)^{i}(-1)^{i+j}(\log \alpha)^{k+1}(j+1)^{k}}{i ! k !(\alpha-1)^{i+1}(k+1)}\left(\begin{array}{l}
i \\
j
\end{array}\right)(k+1)[\bar{H}(x)]^{k}
$$

Where $H(x)$ and $h(x)$ are the DF and the PDF of the baseline distribution respectively. Then (18) can be expressed as

$$
f(x ; \alpha, \theta, \xi)=\sum_{k=0}^{\infty} \varphi_{k} \pi_{k+1}(x)
$$

where

$$
\varphi_{k}=\left[\frac{\theta}{\left(1-e^{-\theta}\right)}\right] \sum_{i, j=0}^{\infty} \frac{(-\theta)^{i}(-1)^{i+j}(\log \alpha)^{k+1}(j+1)^{k}}{i ! k !(\alpha-1)^{i+1}(k+1)}
$$

And

$$
\pi_{k+1}=(k+1) h(x)[H(x)]^{k}
$$

This is the Exp-G PDF with power parameter $(\mathrm{k}+1)$. By integrating (19), we obtain the mixture representation of $\mathrm{F}(\mathrm{x})$ as

$$
F(x ; \alpha, \theta, \xi)=\sum_{k=0}^{\infty} \varphi_{k} \Pi_{k+1}(x)
$$

where $\Pi_{k+1}$ is the cdf of the Exp-G family with power parameter $(k+1)$. Equation (20) reveals that the EGGP density function is a linear combination of Exp-G densities. Thus, some structural properties of the new family such as the ordinary and incomplete moments and the generating function can be immediately obtained from well-established properties of the Exp-G distributions. The properties of Exp-G distributions have been studied by many authors in recent years, see Mudholkar and Srivastava (1993) and Mudholkar, Srivastava and Freimer (1995) for exponentiated Weibull (EW) distributions, R. C. Gupta, P. L. Gupta and R. D. Gupta (1998) for exponentiated Pareto distributions, Gupta and Kundu (1999) for exponentiated exponential distributions, Nadarajah and Kotz (2006) for the exponentiated-type distributions, Nadarajah (2005) for exponentiated Gumbel distributions, Shirke and Kakade (2006) for exponentiated log-normal distributions and Nadarajah and Gupta (2007) for exponentiated gamma distributions (EGa), among others.

\section{Alpha Power Bur XII Poisson Distribution and Properties}

The Bur XII (BXII) distribution was proposed by Burr (1942) has several applications in in many areas including lifetime testing, reliability, failure time modeling and acceptance sampling plans. This distribution is a very popular distribution for modeling lifetime data exhibiting monotone failure rate. Tadikamalla (1980) investigated the properties of Bur XII distribution and its related models, namely: logistic, compound Weibull gamma, Weibull exponential, Pareto type II (Lomax) distributions. In recent time several modifications have been made to BXII distribution to allow for wider applications and this includes: Zimmer et al. (2008) proposed and studied a three-parameter Burr XII distribution; Silva et al. proposed and studied the properties of the log-Burr XII regression models with censored data. Afify et al. (2016) investigated the properties of Weibull BXII; Zografos-Balakrishnan BXII distribution was studied by Altun et al. (2018) distribution and many others. The CDF of Bur XII distribution is given by

$$
\bar{H}(x ; \lambda, \eta)=1-\left(1+x^{\lambda}\right)^{-\eta}, \quad x>0
$$

And the corresponding CDF is given by 


$$
\bar{h}(x ; \lambda, \eta)=\lambda \eta\left(1+x^{\lambda}\right)^{-\eta}, \quad x>0
$$

Where $\lambda$ and $\eta$ are positive shape parameters. Putting (22) in (9), we obtain a CDF of a more flexible distribution named Alpha Power Bur XII Poisson (APBXIIP) distribution given by

$$
F(x ; \alpha, \theta, \lambda, \eta)=\left[\frac{1}{1-e^{-\theta}}\right]\left[1-e^{-\theta\left(\frac{\alpha^{\left(1-\left(1+x^{\lambda}\right)^{-\eta}\right)}}{\alpha-1}\right)}\right]
$$

And the associated $P D F$ is given by

$$
f(x ; \alpha, \theta, \lambda, \eta)=\left[\frac{\lambda \eta \theta \log \alpha}{\left(1-e^{-\theta}\right)(\alpha-1)}\right] x^{\lambda-1}\left(1+x^{\lambda}\right)^{-\eta-1} \alpha^{\left(1-\left(1+x^{\lambda}\right)^{-\eta}\right)} e^{-\theta\left(\frac{\alpha^{\left(1-\left(1+x^{\lambda}\right)^{-\eta}\right)}-1}{\alpha-1}\right)}
$$

Plots of the cdf and PDF of the APBXIIP distribution are displayed in Figure 1 for some parameter values.
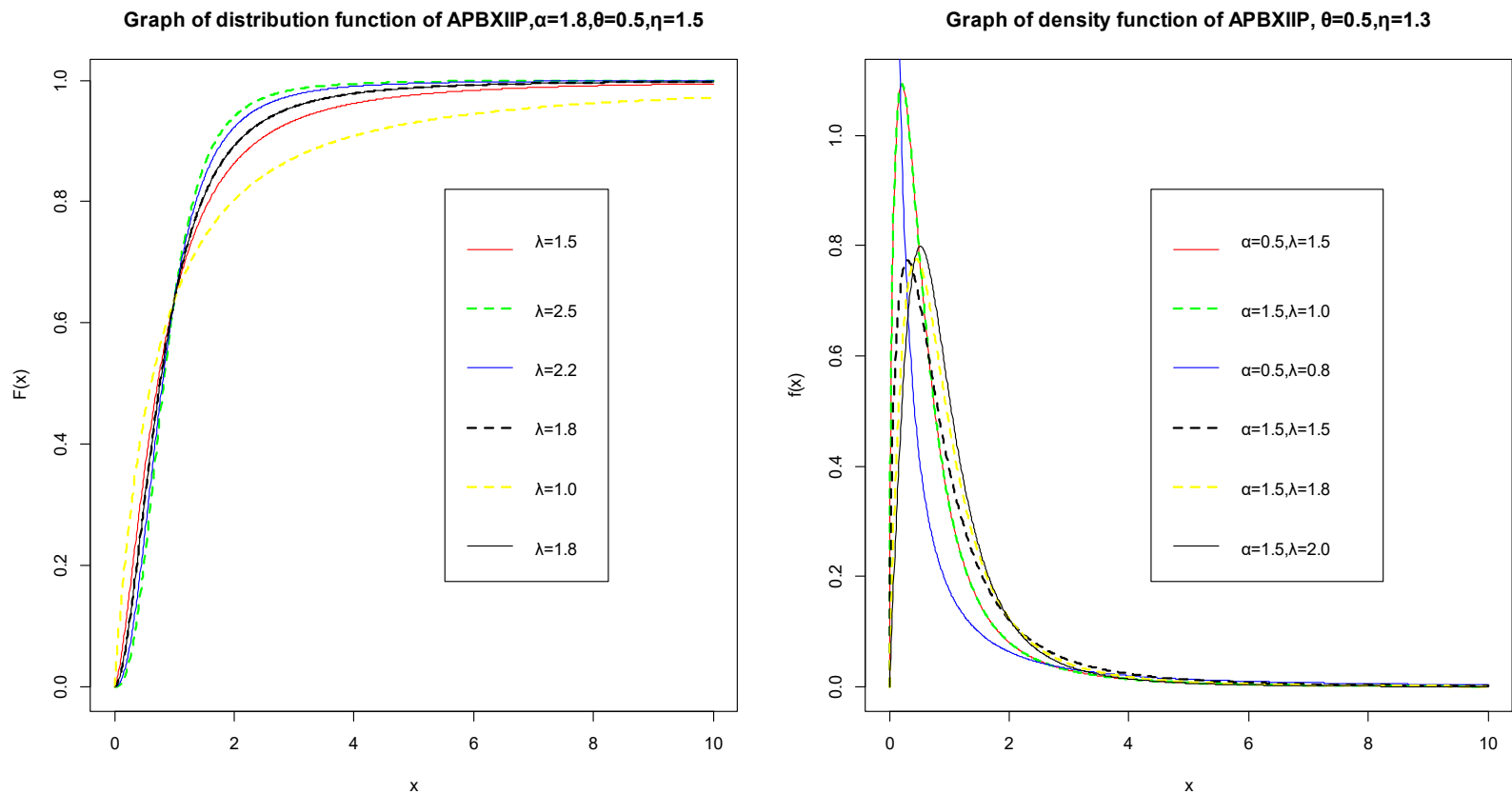

Figure 1. Plot of APBXIIP Distribution and Density Function

An expression for its Survival function $(S(x))$ and hazard function $(h(x))$ is respectively given by

$$
\boldsymbol{S}(\boldsymbol{x})=\mathbf{1}-\left[\frac{1}{1-e^{-\theta}}\right]\left[1-e^{-\theta\left(\frac{\alpha\left(1-\left(1+x^{\lambda}\right)^{-\eta}\right)}{\alpha-1}\right)}\right]
$$

and 


$$
h(x)=\frac{\left[\frac{\lambda \eta \theta \log \alpha}{\left(1-e^{-\theta}\right)(\alpha-1)}\right] x^{\lambda-1}\left(1+x^{\lambda}\right)^{-\eta-1} \alpha^{\left(1-\left(1+x^{\lambda}\right)^{-\eta}\right)} e^{-\theta\left(\frac{\alpha^{\left(1-\left(1+x^{\lambda}\right)^{-\eta}\right)}}{\alpha-1}\right)}}{1-\left[\frac{1}{1-e^{-\theta}}\right]\left[1-e^{-\theta\left(\frac{\alpha^{\left(1-\left(1+x^{\lambda}\right)^{-\eta}\right)}-1}{\alpha-1}\right)}\right]}
$$

Figure (2) and (3) are the graphs of the hazard function of $A P B X I I P$ distribution for various values of the parameters.

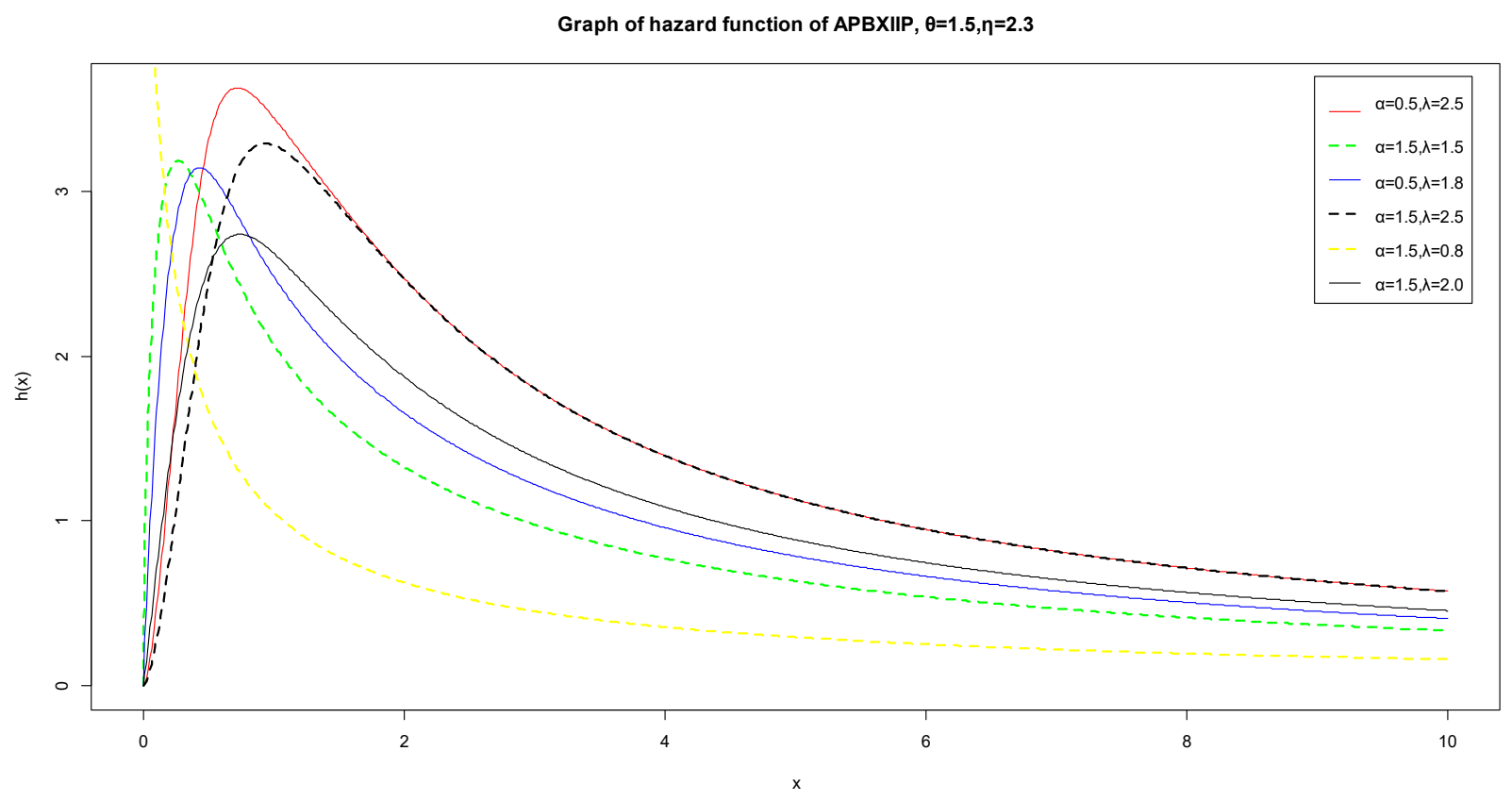

Figure 2. Plot of APBXIIP Hazard Function

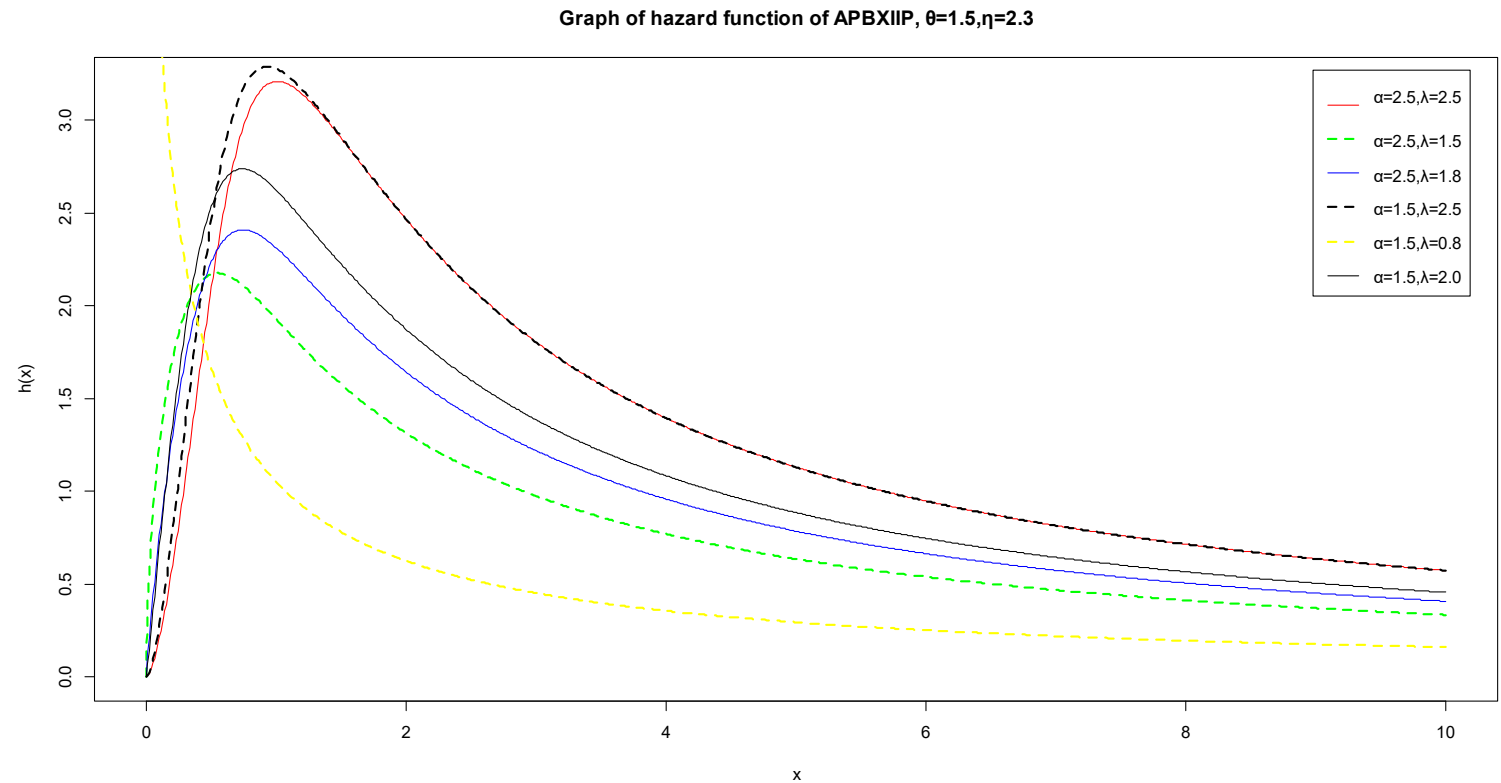

Figure 3. Plot of APBXIIP Hazard Function 
Plot of hazard APBXIIP hazard rate function shows different shapes including decreasing, increasing, as well as inverted (upside down) bathtub shapes as shown in figure (2) and (3).

\subsection{Quantile Function of APBXIIP Model}

The $A P B X I I P$ quantile, say $Q(u)=F^{-1}(u)$, can easily be obtained by inverting The $C D F$ given in (28) as follows:

$$
x_{u}=\left[1-\left(1 / \log (\alpha)\left\{1-\log \left(\frac{\{1-\alpha\}}{\theta}\left[1-u\left(1-e^{-\theta}\right)\right]\right)\right\}\right)^{1 / \lambda}\right]^{1 / \eta},
$$

the $u^{\text {th }}$ quantile for $u \in(0,1)$

for $u=0.25,0.5,0.75$, we have the lower quartile, middle quartile (median) and the upper quartile of the $A P B X I I P$ distribution respectively, given by

$$
\begin{gathered}
x_{0.25}=\left[1-\left(1 / \log (\alpha)\left\{1-\log \left(\frac{\{1-\alpha\}}{\theta}\left[1-0.25\left(1-e^{-\theta}\right)\right]\right)\right\}\right)^{1 / \lambda}\right]^{1 / \eta}, \\
x_{0.5}=\left[1-\left(1 / \log (\alpha)\left\{1-\log \left(\frac{\{1-\alpha\}}{\theta}\left[1-0.5\left(1-e^{-\theta}\right)\right]\right)\right\}\right)^{1 / \lambda}\right]^{1 / \eta},
\end{gathered}
$$

And

$$
x_{0.75}=\left[1-\left(1 / \log (\alpha)\left\{1-\log \left(\frac{\{1-\alpha\}}{\theta}\left[1-0.75\left(1-e^{-\theta}\right)\right]\right)\right\}\right)^{1 / \lambda}\right]^{1 / \eta},
$$

We use the quantile function of $\mathrm{X}$ given in (28) to obtain a numerical value for the Bowley's skewness $\mathrm{B}_{\mathrm{S}}$, Kenny and Keeping (1962) and Moor's kurtosis $\mathrm{M}_{\mathrm{k}}$, Moors (1988). These measures are given by

$$
B_{S}=\frac{Q(3 / 4)+Q(1 / 4)-2 Q(1 / 2)}{Q(3 / 4)-Q(1 / 4)} \text { and } M_{k}=\frac{Q(3 / 8)-Q(1 / 8)+Q(7 / 8)-Q(5 / 8)}{Q(6 / 8)-Q(2 / 8)}
$$

Table 1 drawn below gives numerical values of Bowley's skewness and Moor's kurtosis of APBXIIP model for a fixed value of $\alpha=0.1, \theta=0.2$ and varying the values of $\lambda$ and $\eta$.

Table 1. Table of Bowley's skewness and Moor's kurtosis for APBXIIP model

\begin{tabular}{|c|c|c|c|c|}
\hline \multirow{2}{*}{ Quantiles } & \multicolumn{4}{|c|}{ Parameters } \\
\cline { 2 - 5 } & $\lambda=1.5, \eta=4.7$ & $\lambda=2.5, \eta=217$ & $\lambda=1.5, \eta=0.7$ & $\lambda=0.5, \eta=0.2$ \\
\hline$Q(1 / 4)$ & 0.9192 & 0.6462 & 0.5516 & 0.8175 \\
\hline$Q(1 / 2)$ & 0.9224 & 0.6644 & 0.5812 & 0.8518 \\
\hline$Q(3 / 4)$ & 0.9300 & 0.6846 & 0.6142 & 0.8849 \\
\hline$Q(1 / 8)$ & 0.9117 & 0.6377 & 0.5379 & 0.8000 \\
\hline$Q(3 / 8)$ & 0.9187 & 0.6551 & 0.5661 & 0.8348 \\
\hline$Q(5 / 8)$ & 0.9261 & 0.6742 & 0.5972 & 0.8686 \\
\hline$Q(7 / 8)$ & 0.9340 & 0.6956 & 0.6321 & 0.9006 \\
\hline$B_{S}$ & 0.0270 & 0.0520 & 0.0543 & -0.0178 \\
\hline$M_{k}$ & 1.0068 & 1.0104 & 1.0080 & 0.9911 \\
\hline
\end{tabular}

\subsection{Mixture Representation of $\boldsymbol{A P B X I I P}$ Distribution}

The PDF of $A P B X I I P$ distribution given in (25) can be presented in a mixture form using the expansion series given in (13), (15) and (17) to obtain

$$
f(x)=\frac{\lambda \eta \theta}{\left(1-e^{-\theta}\right)} \sum_{i, j, k, l}^{\infty} \frac{(-1)^{i+j+l}(-\theta)^{i}}{i ! k !(\alpha-1)^{i+1}}\left(\begin{array}{l}
i \\
j
\end{array}\right)\left(\begin{array}{l}
k \\
l
\end{array}\right)(1+j)^{k} x^{\lambda-1}\left(1+x^{\lambda}\right)^{l-\eta-1}
$$




\section{Ordinary and Incomplete Moments of APBXIIP Model}

Several properties of a distribution can be examined via their moments. The $r^{\text {th }}$ moment about the origin of $X$ has a APBXIIP distribution is obtained as follows:

Using the mixture representation of APBXIIP model given in (32), we have

$$
E(X)^{r}=\mu_{r}^{\prime}=\frac{\lambda \eta \theta}{\left(1-e^{-\theta}\right)} \sum_{i, j, k, l}^{\infty} \frac{(-1)^{i+j+l}(-\theta)^{i}}{i ! k !(\alpha-1)^{i+1}}\left(\begin{array}{l}
i \\
j
\end{array}\right)\left(\begin{array}{l}
k \\
l
\end{array}\right)(1+j)^{k} \int_{-\infty}^{\infty} x^{\lambda-1}\left(1+x^{\lambda}\right)^{l-\eta-1} d x
$$

By letting $p=x^{\lambda}, x=p^{1 / \lambda}, d x=1 / \lambda^{1 / \lambda^{-1}} d p$ and putting it in (33), we have

$$
\mu_{r}^{\prime}=\frac{\eta \theta}{\left(1-e^{-\theta}\right)} \sum_{i, j, k, l}^{\infty} \frac{(-1)^{i+j+l}(-\theta)^{i}}{i ! k !(\alpha-1)^{i+1}}\left(\begin{array}{l}
i \\
j
\end{array}\right)\left(\begin{array}{l}
k \\
l
\end{array}\right)(1+j)^{k} \int_{-\infty}^{\infty} p^{r / \lambda}(1+p)^{l-\eta-1} d p
$$

Also, taking $p=1 /(1-u), d p=(1-u)^{-2} d u$ and substitute in (34), we have

$$
\mu_{r}^{\prime}=\frac{\eta \theta}{\left(1-e^{-\theta}\right)} \sum_{i, j, k, l}^{\infty} \frac{(-1)^{i+j+l}(-\theta)^{i}}{i ! k !(\alpha-1)^{i+1}}\left(\begin{array}{l}
i \\
j
\end{array}\right)\left(\begin{array}{l}
k \\
l
\end{array}\right)(1+j)^{k} \int_{-\infty}^{\infty} u^{r} / \lambda(1-u)^{\eta-1-l-\frac{r}{\lambda}} d p
$$

Finally, we have

$$
\mu_{r}^{\prime}=\frac{\eta \theta}{\left(1-e^{-\theta}\right)} \sum_{i, j, k, l}^{\infty} \frac{(-1)^{i+j+l}(-\theta)^{i}}{i ! k !(\alpha-1)^{i+1}}\left(\begin{array}{l}
i \\
j
\end{array}\right)\left(\begin{array}{l}
k \\
l
\end{array}\right)(1+j)^{k} B\left[\left(\frac{r}{\lambda}+1\right),\left(\eta-l-\frac{r}{\lambda}\right)\right]
$$

where $B(q, n)=\int_{0}^{1} z^{q-1}(1-z)^{n-1} d z$, is the standard beta function with $q>0$ and $n>0$.

The mean of APBXIIP distribution can be estimated by taking $r=1$ in equation (), we have

$$
\mu_{1}^{\prime}=\mu=\frac{\eta \theta}{\left(1-e^{-\theta}\right)} \sum_{i, j, k, l}^{\infty} \frac{(-1)^{i+j+l}(-\theta)^{i}}{i ! k !(\alpha-1)^{i+1}}\left(\begin{array}{l}
i \\
j
\end{array}\right)\left(\begin{array}{l}
k \\
l
\end{array}\right)(1+j)^{k} B\left[\left(\frac{1}{\lambda}+1\right),\left(\eta-l-\frac{1}{\lambda}\right)\right]
$$

Table 2 drawn below gives the first six moments, variance $\left(\sigma^{2}\right)$ and the coefficient of variation $(C V)$ taking the values of parameters $\lambda=1.5, \eta=4.1$ and varying the values of $\alpha$ and $\theta$.

The coefficient of variation $(C V)$, variance $\left(\sigma^{2}\right)$, and standard deviation $(S D=\sigma)$ can be easily obtained and are given by

$$
\sigma^{2}=\mu_{2}^{\prime}-\mu^{2}, \text { and } C V=\frac{\sigma}{\mu}=\frac{\left(\mu_{2}^{\prime}-\mu^{2}\right)^{1 / 2}}{\mu}=\left(\frac{\mu_{2}^{\prime}}{\mu^{2}}-1\right)^{1 / 2}
$$

Table 2. Table of moments of APBXIIP Distribution

\begin{tabular}{|c|c|c|c|c|}
\hline Moments & $\alpha=0.1, \theta=0.5$ & $\alpha=1.1, \theta=1.5$ & $\alpha=2.1, \theta=2.5$ & $\alpha=5.0, \theta=5.0$ \\
\hline$\mu_{1}^{\prime}$ & 0.2181 & 0.3003 & 0.2802 & 0.2242 \\
\hline$\mu_{2}^{\prime}$ & 0.0955 & 0.1674 & 0.1420 & 0.0814 \\
\hline$\mu_{3}^{\prime}$ & 0.0769 & 0.1569 & 0.1218 & 0.0472 \\
\hline$\mu_{4}^{\prime}$ & 0.1122 & 0.2468 & 0.1803 & 0.0500 \\
\hline$\mu_{5}^{\prime}$ & 0.3296 & 0.7479 & 0.5300 & 0.1218 \\
\hline$\mu_{6}^{\prime}$ & 6.6154 & 15.1467 & 10.6245 & 2.2960 \\
\hline$\sigma^{2}$ & 0.0479 & 0.0772 & 0.0635 & 0.0311 \\
\hline$C V$ & 1.0035 & 0.9252 & 0.8993 & 0.7865 \\
\hline
\end{tabular}


Further, one can determine the $r^{\text {th }}$ central moment and $r^{\text {th }}$ cumulant of $\mathrm{X}$ defined respectively by,

$$
\mu_{r}=E\left\{(X-\mu)^{r}\right\}=\sum_{q=0}^{r}\left(\begin{array}{l}
r \\
q
\end{array}\right) \mu_{r-q}^{\prime}(-1)^{q} \mu^{q}, \quad \kappa_{r}=\mu_{r}^{\prime}-\sum_{q=1}^{r-1}\left(\begin{array}{l}
r-1 \\
q-1
\end{array}\right) \kappa_{q} \mu_{r-q}^{\prime},
$$

With $\kappa_{1}=\mu$. One can express several measure of skewness and kurtosis based cumulants (central moments) Consequently, the $r^{\text {th }}$ incomplete moment of a distribution is given by

$$
E\left(X^{r}\right)=\int_{t}^{\infty} x^{r} f(x) d x
$$

Putting equation (32) in (38), we obtain

$$
\mu_{s}^{\prime}(t)=\frac{\lambda \eta \theta}{\left(1-e^{-\theta}\right)} \sum_{i, j, k, l}^{\infty} \frac{(-1)^{i+j+l}(-\theta)^{i}}{i ! k !(\alpha-1)^{i+1}}\left(\begin{array}{l}
i \\
j
\end{array}\right)\left(\begin{array}{l}
k \\
l
\end{array}\right)(1+j)^{k} \int_{0}^{\infty} x^{\lambda-1}\left(1+x^{\lambda}\right)^{l-\eta-1} d x
$$

By letting $p=x^{\lambda}, x=p^{1 / \lambda}, d x=1 / \lambda^{1 / \lambda^{-1}} d p$ and putting it in (39), we have

$$
\mu_{r}^{\prime}(t)=\frac{\eta \theta}{\left(1-e^{-\theta}\right)} \sum_{i, j, k, l}^{\infty} \frac{(-1)^{i+j+l}(-\theta)^{i}}{i ! k !(\alpha-1)^{i+1}}\left(\begin{array}{l}
i \\
j
\end{array}\right)\left(\begin{array}{l}
k \\
l
\end{array}\right)(1+j)^{k} \int_{0}^{t^{\lambda}} p^{r / \lambda}(1+p)^{l-\eta-1} d p
$$

Also, taking $p=1 /(1-u), d p=(1-u)^{-2} d u$ and substitute in (40), we have

$$
\mu_{r}^{\prime}=\frac{\eta \theta}{\left(1-e^{-\theta}\right)} \sum_{i, j, k, l}^{\infty} \frac{(-1)^{i+j+l}(-\theta)^{i}}{i ! k !(\alpha-1)^{i+1}}\left(\begin{array}{l}
i \\
j
\end{array}\right)\left(\begin{array}{l}
k \\
l
\end{array}\right)(1+j)^{k} \int_{0}^{\frac{t^{\lambda}}{\left(1+t^{\lambda}\right)}} u^{r / \lambda}(1-u)^{\eta-1-l-\frac{r}{\lambda}} d p
$$

Finally, we have

$$
\mu_{r}^{\prime}=\frac{\eta \theta}{\left(1-e^{-\theta}\right)} \sum_{i, j, k, l}^{\infty} \frac{(-1)^{i+j+l}(-\theta)^{i}}{i ! k !(\alpha-1)^{i+1}}\left(\begin{array}{l}
i \\
j
\end{array}\right)\left(\begin{array}{l}
k \\
l
\end{array}\right)(1+j)^{k} B\left[\frac{t^{\lambda}}{\left(1+t^{\lambda}\right)},\left(\frac{r}{\lambda}+1\right),\left(\eta-l-\frac{r}{\lambda}\right)\right]
$$

where $B(z ; q, n)=\int_{0}^{z} y^{z-1}(1-y)^{n-1} d y$, is the beta function

By taking $r=1$, we obtain an expression for the first incomplete moment as

$$
\mu_{1}^{\prime}=\frac{\eta \theta}{\left(1-e^{-\theta}\right)} \sum_{i, j, k, l}^{\infty} \frac{(-1)^{i+j+l}(-\theta)^{i}}{i ! k !(\alpha-1)^{i+1}}\left(\begin{array}{l}
i \\
j
\end{array}\right)\left(\begin{array}{l}
k \\
l
\end{array}\right)(1+j)^{k} B\left[\frac{t^{\lambda}}{\left(1+t^{\lambda}\right)},\left(\frac{1}{\lambda}+1\right),\left(\eta-l-\frac{1}{\lambda}\right)\right]
$$

It should be noted that $\mu_{r}^{\prime}(t)$ always exists.

\subsection{Moment Generating Function}

The moment generating function of a random variable $X$ is defined by

$$
E\left(e^{t X}\right)=\int_{-\infty}^{\infty} e^{t X} f(x) d x
$$




$$
\begin{aligned}
=\sum_{r=0}^{\infty} \frac{t^{r}}{r !} \int_{0}^{\infty} x^{r} f(x) d x \\
\quad=\sum_{i, j, k, l, r}^{\infty} \frac{t^{r}}{r !} \frac{\eta \theta}{\left(1-e^{-\theta}\right)} \frac{(-\theta)^{i}(-1)^{i+j+l}}{i ! k !(\alpha-1)^{i+1}}\left(\begin{array}{l}
i \\
j
\end{array}\right)\left(\begin{array}{l}
k \\
l
\end{array}\right)(1+j)^{k} B\left[\left(\frac{r}{\lambda}+1\right),\left(\eta-l-\frac{r}{\lambda}\right)\right]
\end{aligned}
$$

\subsection{Mean Deviation}

The mean deviation, about the mean and the median, are used to determine the degree of spread in a population. Let $\mu$ and $M$ be the mean and the median of the APBXIIP distribution given by (43) and (30) respectively.

The mean deviation of $A P B X I I P$ model about the mean can be obtained as

$$
\begin{aligned}
& \Gamma_{1}(X)=E|X-\mu|=\int_{0}^{\infty}|X-\mu| f(x ; \alpha, \theta, \lambda, \eta) d x \\
& =2 \mu F(\mu ; \alpha, \theta, \lambda, \eta)-2 \mu+2 \int_{\mu}^{\infty} x f(x ; \alpha, \theta, \lambda, \eta) d x \\
& =2 \mu\left[\frac{1}{1-e^{-\theta}}\right]\left[\begin{array}{l}
-\theta\left(\frac{\alpha^{\left(1-\left(1+x^{\lambda}\right)^{-\eta}\right)}-1}{\alpha-1}\right) \\
1-e^{-1}
\end{array}\right]-2 \mu+\frac{2 \eta \theta}{\left(1-e^{-\theta}\right)} \\
& \times \sum_{i, j, k, l}^{\infty} \frac{(-1)^{i+j+l}}{i ! k !(\alpha-1)^{i+1}}\left(\begin{array}{l}
i \\
j
\end{array}\right)\left(\begin{array}{l}
k \\
l
\end{array}\right)(1+j)^{k} B\left[\frac{\mu^{\lambda}}{\left(1+\mu^{\lambda}\right)},\left(\frac{r}{\lambda}+1\right),\left(\eta-l-\frac{r}{\lambda}\right)\right]
\end{aligned}
$$

The mean deviation of APBXIIP about the median can also be obtained as

$$
\begin{gathered}
\Gamma_{2}(X)=E|X-M|=\int_{0}^{\infty}|X-M| f(x) d x, \\
=-\mu+2 \int_{m}^{\infty} x f(x ; v, w, \lambda) d x \\
=-\boldsymbol{\mu}+\frac{2 \eta \theta}{\left(1-e^{-\theta}\right)} \sum_{i, j, k, l}^{\infty} \frac{(-\theta)^{i}(-1)^{i+j+l}}{i ! k !(\alpha-1)^{i+1}}\left(\begin{array}{l}
i \\
j
\end{array}\right)\left(\begin{array}{l}
k \\
l
\end{array}\right)(1+j)^{k} B\left[\frac{m^{\lambda}}{\left(1+m^{\lambda}\right)},\left(\frac{r}{\lambda}+1\right),\left(\eta-l-\frac{r}{\lambda}\right)\right]
\end{gathered}
$$

\subsection{Bonferroni and Lorenz Curves}

The Bonferroni and Lorenz curves have been found suitable to study income and poverty analysis in the field of economics and also in other field like insurance, reliability, and demography. The Bonferroni and Lorenz curves are defined by

$$
B(f)=\frac{1}{p \mu} \int_{0}^{q} x f(x ; \alpha, \theta, \lambda, \eta) d x
$$

and

$$
L(f)=\frac{1}{\mu} \int_{0}^{q} x f(x ; \alpha, \theta, \lambda, \eta) d x
$$


Respectively, where $\mu=E(X)$ and $q=F^{-1}(p)$. In the case of $A P B X I I P$ distribution, we obtain

$$
B(f)=\frac{\theta \eta}{f \mu\left(1-e^{-\theta}\right)} \sum_{i, j, k, l}^{\infty} \frac{(-\theta)^{i}(-1)^{i+j+l}}{i ! k !(\alpha-1)^{i+1}}\left(\begin{array}{l}
i \\
j
\end{array}\right)\left(\begin{array}{l}
k \\
l
\end{array}\right)(1+j)^{k} B\left[\frac{t^{\lambda}}{\left(1+t^{\lambda}\right)} ;\left(\frac{r}{\lambda}+1\right),\left(\eta-l-\frac{r}{\lambda}\right)\right]
$$

and

$$
\begin{gathered}
L(p)=\frac{\eta \theta}{\mu\left(1-e^{-\theta}\right)} \sum_{i, j, k, l}^{\infty} \frac{(-\theta)^{i}(-1)^{i+j+l}}{i ! k !(\alpha-1)^{i+1}}\left(\begin{array}{l}
i \\
j
\end{array}\right)\left(\begin{array}{l}
k \\
l
\end{array}\right)(1+j)^{k} B\left[\frac{t^{\lambda}}{\left(1+t^{\lambda}\right)} ;\left(\frac{r}{\lambda}+1\right),\left(\eta-l-\frac{r}{\lambda}\right)\right] \\
\text { where } B(l ; m, n)=\int_{0}^{l} y^{m-1}(1-y)^{n-1} d y, \text { is the beta function }
\end{gathered}
$$

\section{Renyi Entropy}

Renyi (1961), gave a useful mathematical expression that can be used to measure the entropy of a $A P B X I I P$ distribution given by

$$
I_{R}^{(v)}=\frac{1}{1-v} \log \left[\int_{0}^{\infty} f_{\mathrm{APBXIIP}}(x ; \zeta)^{v} d x\right], \quad v>0, v \neq 1
$$

Putting equation (25) in (52), we have

$$
I_{R}^{(v)}=\frac{1}{1-v} \log \left(W^{v}\right)
$$

Where,

$$
W^{v}=\left[\int_{0}^{\infty}\left\{\left[\frac{\lambda \eta \theta \log \alpha}{\left(1-e^{-\theta}\right)(\alpha-1)}\right] x^{\lambda-1}\left(1+x^{\lambda}\right)^{-\eta-1} \alpha^{\left(1-\left(1+x^{\lambda}\right)^{-\eta}\right)} e^{-\theta\left(\frac{\alpha^{\left(1-\left(1+x^{\lambda}\right)^{-\eta}\right)}-1}{\alpha-1}\right)}\right\} d x\right]
$$

Using series expansion given in (13), (15), and (17) in (53), we have

\section{Order Statistics}

$$
\begin{gathered}
W^{v}=\frac{\lambda^{v-1} \eta^{v} \theta^{v}}{\left(1-e^{-\theta}\right)^{v}(\alpha-1)^{v}} \sum_{i, j, k, l}^{\infty} \frac{(-v \theta)^{i}}{i ! k !}(\log (\alpha))^{k+v}(-1)^{i+j+l}\left(\begin{array}{l}
i \\
j
\end{array}\right)\left(\begin{array}{l}
k \\
l
\end{array}\right)(v+j)^{k} \\
\times B\left[\frac{V(\lambda-1)+1}{\lambda}, \frac{v(\lambda \eta-2 \lambda+1)+l \lambda \eta-1}{\lambda}\right]
\end{gathered}
$$

The concept of order statistics is generally applied in modeling some certain random system. Most especially, for $r=1, \ldots, n$, the first order statistics of a statistical sample is obtained when $r=1$ and for the largest order statistics is when $r=n$.

Suppose $X_{(1)}, X_{(2)}, \ldots, X_{(n)}$ be an ordered sample that follows the APBXIIP distribution, the $p d f$ of $X_{(r)}$ is computed as

$$
f_{r}(x ; \xi)=\frac{1}{B(r, n-r+1)} F_{A P B X I I P}(x ; \xi)^{r-1}\left[1-F_{A P B X I I P}(x ; \xi)\right]^{n-r} f_{A P B X I I P}(x ; \zeta)
$$

Then by applying the series expansion given in (15) to (55), we have 


$$
f_{r}(x ; \xi)=\frac{f_{A P B X I I P}(x ; \xi)}{B(r, n-r+1)} \sum_{i=1}^{n-r}(-1)^{i}\left(\begin{array}{c}
n-r \\
i
\end{array}\right) F_{A P B X I I P}(x ; \xi)^{r+i-1}
$$

Now, by substituting equation (9) and (10) in $f_{r}(x ; \xi)$, we have

$$
\begin{gathered}
f_{r}(x ; \xi)=\frac{\left[\frac{\lambda \eta \theta \log \alpha}{\left(1-e^{-\theta}\right)(\alpha-1)}\right] x^{\lambda-1}\left(1+x^{\lambda}\right)^{-\eta-1} \alpha^{\left(1-\left(1+x^{\lambda}\right)^{-\eta}\right)} e^{-\theta\left(\frac{\alpha^{\left(1-\left(1+x^{\lambda}\right)^{-\eta}\right)}-1}{\alpha-1}\right)}}{B(r, n-r+1)} \\
\left.\times \sum_{i=1}^{n-r}(-1)^{i}\left(\begin{array}{c}
n-r \\
i
\end{array}\right)\left\{\left[\frac{1}{1-e^{-\theta}}\right]\left[1-e^{-\theta\left(\frac{\alpha^{\left(1-\left(1+x^{\lambda}\right)^{-\eta}\right)}-1}{\alpha-1}\right)}\right)\right]\right\}
\end{gathered}
$$

Using the series expansion given in (15) in (57)

$$
\left\{\left[1-e^{-\theta\left(\frac{\alpha^{\left(1-\left(1+x^{\lambda}\right)^{-\eta}\right)}-1}{\alpha-1}\right)}\right]\right\}^{r+i-1}=\sum_{j=0}^{r+i-1}(-1)^{j}\left(\begin{array}{c}
r+i-1 \\
j
\end{array}\right) e^{-\theta j\left(\frac{\alpha^{\left(1-\left(1+x^{\lambda}\right)^{-\eta}\right)}-1}{\alpha-1}\right)}
$$

It then follows that,

$$
\begin{aligned}
& f_{r}(x ; \xi)=\frac{\lambda \eta \theta \log \alpha}{(\alpha-1) B(r, n-r+1)} x^{\lambda-1}\left(1+x^{\lambda}\right)^{-\eta-1} \alpha^{\left(1-\left(1+x^{\lambda}\right)^{-\eta}\right)} \\
& \times \sum_{i=0}^{n-r} \sum_{j=0}^{r+i-1}(-1)^{i+j}\left(\begin{array}{c}
n-r \\
i
\end{array}\right)\left(\begin{array}{c}
r+i-1 \\
j
\end{array}\right)\left(\frac{1}{1-e^{-\theta}}\right)^{r+i} e^{-\theta(j+1)\left(\frac{\alpha^{\left(1-\left(1+x^{\lambda}\right)^{-\eta}\right)}-1}{\alpha-1}\right)}
\end{aligned}
$$

Also, applying (13) and (17) in (58), we have an expression for the $r^{\text {th }}$ order statistics of APBXIIP distribution given by

$$
\begin{aligned}
f_{r}(x ; \xi) & =\frac{\lambda \eta \theta}{B(r, n-r+1)} \sum_{i=0}^{n-r} \sum_{j=0}^{n+r-1} \sum_{k, l, p}^{\infty} \frac{(-1)^{i+j+k+p}}{(\alpha-1)^{k+1} m !}\left(\begin{array}{c}
n-r \\
i
\end{array}\right)\left(\begin{array}{c}
r+i-1 \\
j
\end{array}\right)\left(\begin{array}{c}
k \\
l
\end{array}\right)\left(\begin{array}{c}
m \\
p
\end{array}\right) \\
& \times[\log (\alpha)]^{m+1}\left(\frac{1}{1-e^{-\theta}}\right)^{r+i}[-\theta(j+1)]^{k}(m+1)^{k} x^{\lambda-1}\left(1+x^{\lambda}\right)^{-[\eta(1+p)+1]}
\end{aligned}
$$

It should be noted that Renyi entropy is an extension of Shannon entropy that Renyi entropy tends to Shannon entropy as $v \rightarrow 1$

\section{Maximum Likelihood Estimates of the Parameters}

Estimators are obtained for the APBXIIP parameters depending on the maximum likelihood estimates are derived. Suppose $X_{1}, X_{2}, \ldots, X_{n}$ be a random sample from APBXIIP distribution with observed values $x_{1}, x_{2}, \ldots, x_{n}$. The log likelihood function of APBXIIP model, denoted by $l$, is obtained as follows

$$
\begin{aligned}
l=n \log \left[\frac{\lambda \eta \theta \log \alpha}{\left(1-e^{-\theta}\right)(\alpha-1)}\right]+(\lambda-1) \sum_{i=1}^{n} x_{i}-(\eta+1) \sum_{i=1}^{n}\left(1+x_{i}^{\lambda}\right) \\
\quad+\log (\alpha) \sum_{i=1}^{n}\left(1-\left(1+x_{i}^{\lambda}\right)^{-\eta}\right)-\frac{\theta}{(\alpha-1)} \sum_{i=1}^{n}\left[\alpha^{\left(1-\left(1+x^{\lambda}\right)^{-\eta}\right)}-1\right]
\end{aligned}
$$

The partial derivative of the log-likelihood function with respect to the unknown parameters are given by 


$$
\begin{aligned}
& \frac{\partial l}{\partial \alpha}=\frac{n(\alpha-1-\alpha \log (\alpha))}{\alpha(\alpha-1) \log (\alpha)}+\frac{1}{\alpha} \sum_{i=1}^{n}\left(1-\left(1+x_{i}^{\lambda}\right)^{-\eta}\right)+\frac{1}{(\alpha-1)^{2}} \sum_{i=1}^{n}\left[\alpha^{\left(1-\left(1+x_{i}^{\lambda}\right)^{-\eta}\right)}-1\right] \\
& \frac{\partial l}{\partial \theta}=\frac{n}{\theta}-\frac{n e^{-\theta}}{1-e^{-\theta}}-\frac{1}{(\alpha-1)} \sum_{i=1}^{n}\left[\alpha\left(1-\left(1+x^{\lambda}\right)^{-\eta}\right)-1\right] \\
& -\frac{1}{\alpha(\alpha-1)} \sum_{i=1}^{n} \alpha^{\left(1-\left(1+x_{i}^{\lambda}\right)^{-\eta}\right)}\left(1-\left(1+x_{i}^{\lambda}\right)^{-\eta}\right) \\
& \frac{\partial l}{\partial \lambda}=\frac{n}{\lambda}+\sum_{i=1}^{n} x_{i}-(\eta+1) \sum_{i=1}^{n} x_{i}^{\lambda} \log (x)+\eta \log (\alpha) \sum_{i=1}^{n} x_{i}^{\lambda} \log \left(x_{i}\right)\left(1+x_{i}^{\lambda}\right)^{-\eta-1} \\
& -\frac{\theta \eta \log (\alpha)}{(\alpha-1)} \sum_{i=1}^{n} x_{i}^{\lambda}\left(1+x_{i}^{\lambda}\right)^{-\eta-1} \log \left(x_{i}\right) \\
& \frac{\partial l}{\partial \eta}=\frac{n}{\eta}-\sum_{i=1}^{n}\left(1+x_{i}^{\lambda}\right)-\log (\alpha) \sum_{i=1}^{n}\left(1+x_{i}^{\lambda}\right)^{-\eta} \log \left(1+x_{i}^{\lambda}\right)
\end{aligned}
$$

The maximum likelihood of the model parameters are obtained by solving the non-linear equations $\frac{\partial l}{\partial \alpha}=\frac{\partial l}{\partial \theta}=\frac{\partial l}{\partial \lambda}=$ $\frac{\partial l}{\partial \eta}=0$. The solutions to these equations can be obtained by solving simultaneously, numerically using iterative method such as Newton-Raphson iteration technique. For interval estimation of the parameters, the $4 \times 4$ observed information matrix $I(\xi)=\left\{I_{q p}\right\}$ for $(\alpha, \theta, \lambda, \eta)$. U7nder certain regularity conditions, the asymptotic properties of the maximum likelihood method shows that: $\sqrt{n}(\hat{\xi}-\xi) \stackrel{d}{\rightarrow}$ indicates the convergence in distribution, with mean 0. Then the $100(1-w) \%$ confidence intervals for $\alpha, \theta, \lambda$ and $\eta$ are given respectively as follows:

$$
\hat{\alpha} \pm Z_{w} S E(\hat{\alpha}), \hat{\theta} \pm Z_{w} S E(\hat{\theta}), \hat{\lambda} \pm Z_{w} S E(\hat{\lambda}) \text { and } \hat{\eta} \pm Z_{w} S E(\hat{\eta})
$$

It should be noted that the variances of $\alpha, \theta, \lambda, \eta$ are the diagonal elements of $I^{-1}(\xi)$ corresponding to the model parameters.

\section{Simulation Study}

A simulation study is carried to examine the performance of MLE for APBXIIP model in terms of their absolute bias $(\mathrm{AB})$, mean square error (MSE). In this context, we employ of the most used simulation techniques to evaluate the performance of estimators is by Monte Carlo simulation, see, for example Lemonte (2013), Cordeiro and Lemonte (2014) and De Andrade et al. (2019) Simulated procedures are carried out as follows:

A sample sizes of $n=50,100,150$ and 200 are generated from APBXIIP distribution with selected values for $\alpha, \theta, \lambda$ and $\eta$. We consider 2000 Monte Carlo replications. The simulation process is performed in $\mathrm{R}$ software using Broyden-Fletcher-Goldfarb-Shannon (BFGS) maximization method in the optimum script. To ensure that the experiment is reproducible, we use the seed for the random number generator: set.seed (103). The results of the simulation are presented in Table 3, including the Absolute mean (AB), standard error (SE) and the mean square error. The results obtained shows that the APBXIIP estimates exhibits desirable properties even for small sample sizes. In general, the MSE approaches zero as the sample size increases, as expected. 
Table 3. Means, Absolute Biases and MSE of $\hat{\alpha}, \hat{\theta}, \hat{\lambda}$, and $\hat{\eta}$ for APBXII model

\begin{tabular}{|c|c|c|c|c|}
\hline Parameter & $n$ & $A B$ & $S E$ & $M S E$ \\
\hline \multirow{5}{*}{$\alpha=0.1$} & 50 & 3.6699 & 3.8566 & 28.3349 \\
\hline & 100 & 5.1857 & 5.4407 & 56.4927 \\
\hline & 150 & 4.0680 & 2.4804 & 22.7010 \\
\hline & 200 & 3.1195 & 1.8514 & 13.1590 \\
\hline & 250 & 2.9146 & 1.3805 & 10.4007 \\
\hline \multirow{5}{*}{$\theta=0.5$} & 50 & 1.7670 & 0.9618 & 4.0474 \\
\hline & 100 & 1.5682 & 0.7460 & 3.0158 \\
\hline & 150 & 1.5337 & 0.5967 & 2.7083 \\
\hline & 200 & 1.6821 & 0.5355 & 3.1162 \\
\hline & 250 & 1.5481 & 0.4613 & 3.1162 \\
\hline \multirow{5}{*}{$\lambda=1.2$} & 50 & 0.4173 & 0.1312 & 2.6094 \\
\hline & 100 & 0.3852 & 0.1072 & 0.1599 \\
\hline & 150 & 0.4536 & 0.0749 & 0.2114 \\
\hline & 200 & 0.3969 & 0.0715 & 0.1626 \\
\hline & 250 & 0.3606 & 0.0646 & 0.1342 \\
\hline \multirow{5}{*}{$\eta=0.5$} & 50 & 1.2080 & 1.0521 & 2.5662 \\
\hline & 100 & 0.8161 & 0.8212 & 1.3404 \\
\hline & 150 & 1.3779 & 0.6358 & 2.3028 \\
\hline & 200 & 0.5556 & 0.5860 & 0.6521 \\
\hline & 250 & 0.5149 & 0.5407 & 0.5575 \\
\hline
\end{tabular}

\subsection{Real Data Applications}

To demonstrate the flexibility proposed family of distributions, $-2 * \log$-likelihood statistic $(-2 l)$, Akaike information criterion $(A I C=2 k-2 l)$, Bayesian information criterion $(B I C=k \ln (n)-2 l)$, Consistent Akaike information criterion $\left(C A I C=A I C+2 \frac{k(k+1)}{n-k-1}\right)$ and Hannan-Quinn information criterion (HQIC) are calculated for APBXIIP model and its sub-models, where $n$ is the number of observations, and $k$ is the number of estimated parameters. The goodness-of-fit statistic, Anderson-Darling $\left(A^{*}\right)$ and Cramer-von Mises $(W *)$ are also presented in the Table. The best model correspond among the class considered is the model having minimum value of these statistics as the best model. In this study, numerical results (of maximum likelihood estimates and goodness of fit criteria) are calculated by using the goodness.fit (.) command in the Model Adequacy package available in R language. The AIC, CAIC, BIC, HQIC, $A^{*}$, and $W^{*}$ are given for the sub-models Alpha Power BXII (APBXII) model, Bur XII Poisson $(B X I I P)$ model and the BXII model. Tables 6, 8 and 10 respectively. The PDF of APBXIIP sub-models are given as

$$
\begin{gathered}
f_{A P B X I I}(x ; \alpha, \theta, \lambda, \eta)=\frac{\lambda \eta \log \alpha}{(\alpha-1)} x^{\lambda-1}\left(1+x^{\lambda}\right)^{-\eta-1} \alpha^{\left(1-\left(1+x^{\lambda}\right)^{-\eta}\right)}, x>0 ; \lambda, \eta, \alpha>0 \\
f_{B X I I P}(x ; \alpha, \theta, \lambda, \eta)=\frac{\lambda \eta \theta}{\left(1-e^{-\theta}\right)} x^{\lambda-1}\left(1+x^{\lambda}\right)^{-\eta-1} e^{-\theta\left(1-\left(1+x^{\lambda}\right)^{-\eta}\right)}, x>0 ; \theta, \eta, \alpha>0
\end{gathered}
$$

The following are the data sets which we have used in this study. Data set 1 represents the lifetime of 50 devices and was used by Aarset (1987).

Data set 1: $0.1,0.2,1,1,1,1,1,2,3,6,7,11,12,18,18,18,18,18,21,32,36,40,45,46,47,50,55,60,63,63,67,67$, $67,67,72,75,79,82,82,83,84,84,84,85,85,85,85,85,86,86$.

Data set 2 contains intervals in days between 109 successive coal-mining disasters in Great Britain, for the period 18751951, published by Mahdavi and Kundu (2017). The data are given as:

Data set $2: 1,4,4,7,11,13,15,15,17,18,19,19,20,20,22,23,28,29,31,32,36,37,47,48,49,50,54,54,55,59$, $59,61,61,66,72,72,75,78,78,81,93,96,99,108,113,114,120,120,120,123,124,129,131,137,145,151,156$, $171,176,182,188,189,195,203,208,215,217,217,217,224,228,233,255,271,275,275,275,286,291,312,312$, $312,315,326,326,329,330,336,338,345,348,354,361,364,369,378,390,457,467,498,517,566,644,745,871$, 
$1312,1357,1613,1630$.

Data set 3 contains the remission times (in months) of a random sample of 128 bladder cancer patients. The data have been obtained from Lee and Wang (2003).

The data are given as: $0.08,2.09,3.48,4.87,6.94,8.66,13.11,23.63,0.20,2.23,3.52,4.98,6.97,9.02,13.29,0.40$, $2.26,3.57,5.06,7.09,9.22,13.80,25.74,0.50,2.46,3.64,5.09,7.26,9.47,14.24,25.82,0.51,2.54,3.70,5.17,7.28$, $9.74,14.76,26.31,0.81,2.62,3.82,5.32,7.32,10.06,14.77,32.15,2.64,3.88,5.32,7.39,10.34,14.83,34.26,0.90$, $2.69,4.18,5.34,7.59,10.66,15.96,36.66,1.05,2.69,4.23,5.41,7.62,10.75,16.62,43.01,1.19,2.75,4.26,5.41,7.63$, $17.12,46.12,1.26,2.83,4.33,5.49,7.66,11.25,17.14,79.05,1.35,2.87,5.62,7.87,11.64,17.36,1.40,3.02,4.34,5.71$, $7.93,11.79,18.10,1.46,4.40,5.85,8.26,11.98,19.13,1.76,3.25,4.50,6.25,8.37,12.02,2.02,3.31,4.51,6.54$, $8.53,12.03,20.28,2.02,3.36,6.76,12.07,21.73,2.07,3.36,6.93,8.65,12.63,22.69$.

Some descriptive statistics for the three data sets considered are presented in Table 4, including the range, mean, median, upper and lower quartile, and variance, among others. From the Table it can deduce that the three data sets are over-dispersed. The graph of Total Test Time (TTT curves) to this data sets are presented in Figure 5, which shows that data set 1 and 3 exhibits non-monotone failure rate and data set 2 exhibits a decreasing failure rate. The graph of empirical density is drawn in figure 4 which shows that data set 1 is moderately skewed to the right and data set 2 and 3 are highly skewed to the right.

Table 4. Descriptive statistics for the data sets

\begin{tabular}{|c|c|c|c|}
\hline Statistic & Data set I & Data set 2 & Data set 3 \\
\hline$n$ & 50 & 109 & 128 \\
\hline Lower quartile & 13.50 & 54.0 & 3.35 \\
\hline Upper quartile & 81.25 & 312.0 & 11.84 \\
\hline Median & 48.50 & 145.0 & 6.40 \\
\hline Mean & 45.69 & 233.3 & 9.37 \\
\hline minimum & 0.1 & 1.0 & 0.08 \\
\hline Maximum & 86.00 & 1630.0 & 79.05 \\
\hline Variance & 1078.16 & 87873.33 & 110.43 \\
\hline range & 86.1 & 1630.1 & 79.13 \\
\hline
\end{tabular}

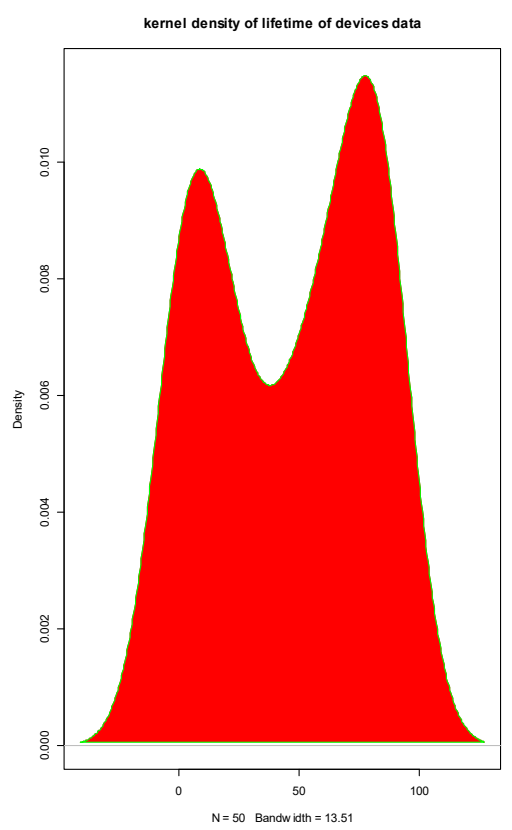

Diagram 1

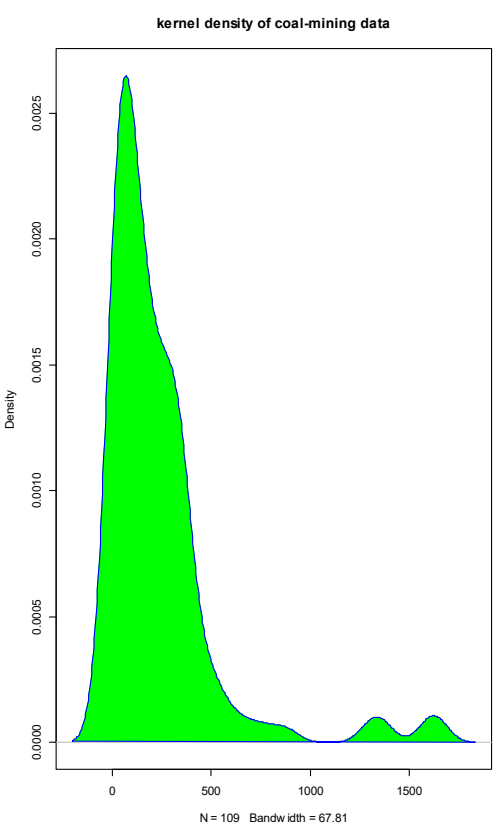

Diagram 2

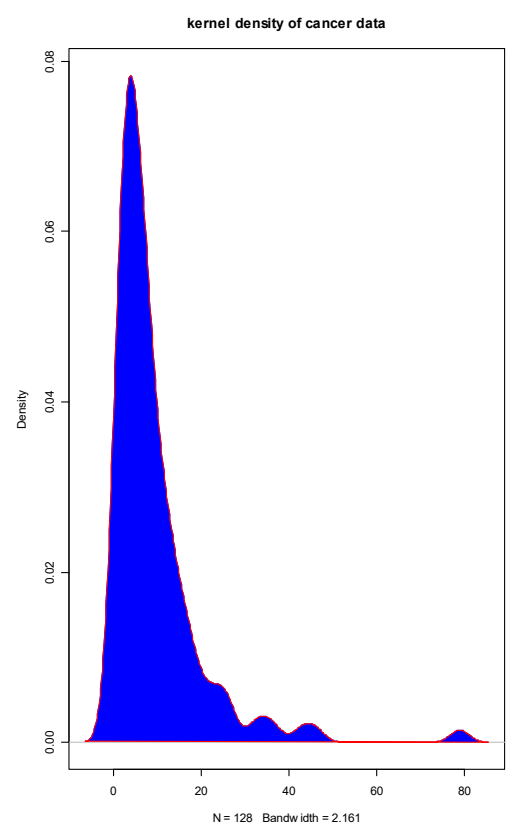

Diagram 3

Figure 4. Kernel density Plot for the three failure data 

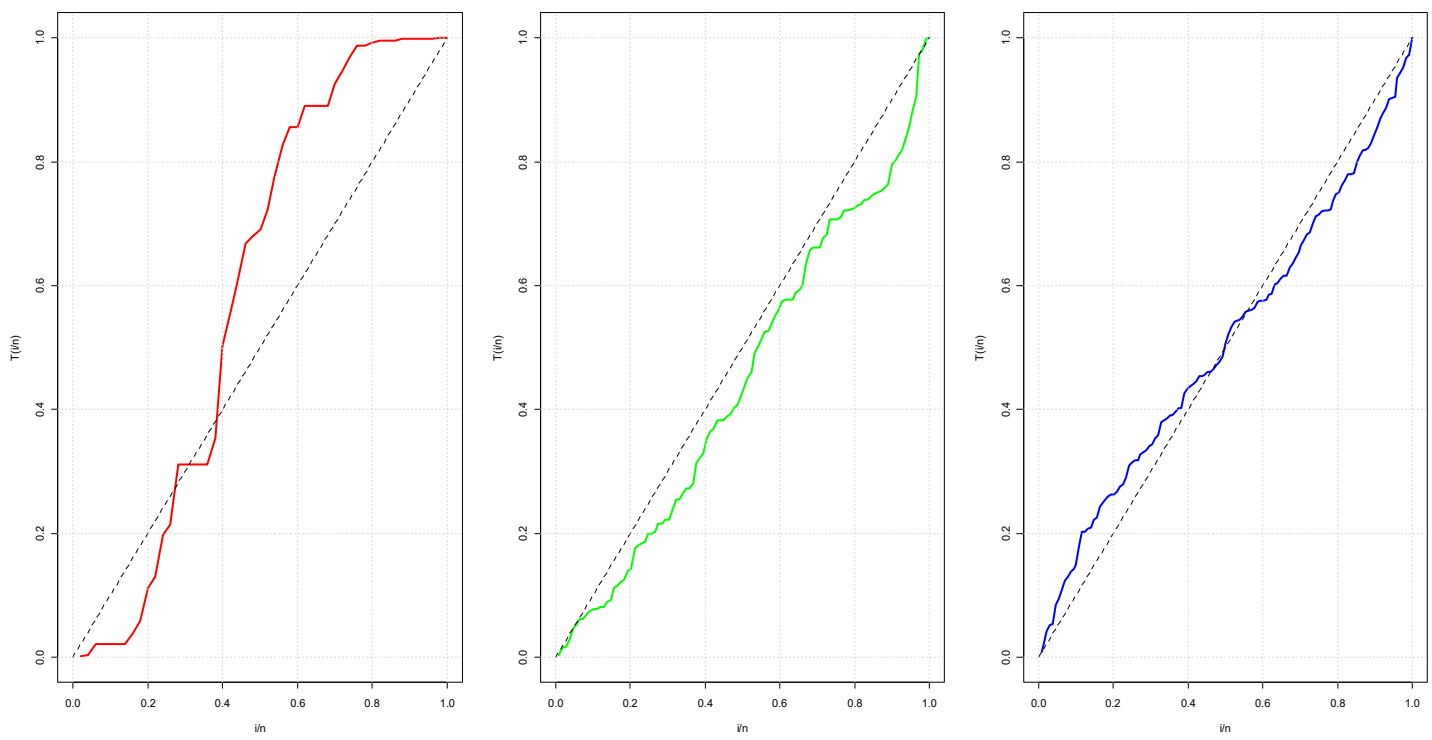

Diagram I

Diagram II

Diagram III

Figure 5. TTT Plot of the three failure data

Table 5. MLEs and SEs (in Parenthesis), confidence interval (in curly bracket) for the device lifetime data

\begin{tabular}{|c|c|c|c|c|}
\hline Distribution & $\alpha$ & $\theta$ & $\lambda$ & $\eta$ \\
\hline APBXIIP & $10.28(1.96)$ & $-4.45(1.77)$ & $0.58(0.17)$ & $1.38(0.46)$ \\
& $\{6.44,14.12\}$ & $\{-7.92,-0.98\}$ & $\{0.25\}$ & $\{0.48,2.28\}$ \\
\hline APBXII & $21.61(12.21)$ & $-(-)$ & $0.98(0.29)$ & $0.56(0.19)$ \\
& $\{-2.32,45.54\}$ & $\{-\}$ & $\{0.42,1.55\}$ & $\{0.19,0.93\}$ \\
\hline PBXII & $-(-)$ & $-8.71(4.76)$ & $0.48(0.20)$ & $1.53(0.79)$ \\
& $\{-\}$ & $\{-18.04,0.62\}$ & $\{0.09,0.87\}$ & $\{-0.02,3.08\}$ \\
\hline BXII & $-(-)$ & $-(-)$ & $1.26(0.32)$ & $0.25(0.07)$ \\
& $\{-\}$ & $\{-\}$ & $\{0.63,1.89\}$ & $\{0.11,0.39\}$ \\
\hline
\end{tabular}

Table 6. The AIC, BIC, CAIC, and $A^{*}, W^{*}$ statistics for device lifetime data

\begin{tabular}{|c|c|c|c|c|l|l|l|}
\hline Distribution & $-2 l$ & AIC & BIC & CAIC & HQIC & $A^{*}$ & \multicolumn{1}{|c|}{$W^{*}$} \\
\hline$A P B X I I P$ & 510.019 & 518.019 & 525.668 & 518.908 & 520.932 & 4.5035 & 0.7858 \\
\hline$A P B X I I$ & 521.757 & 527.757 & 533.493 & 528.279 & 529.942 & 5.0513 & 0.9090 \\
\hline PBXII & 514.864 & 520.864 & 526.599 & 521.386 & 523.048 & 4.8438 & 0.8645 \\
\hline BXII & 544.728 & 548.728 & 552.553 & 548.983 & 550.184 & 5.8572 & 1.0947 \\
\hline
\end{tabular}

Table 7. MLEs and SEs (in Parenthesis), confidence interval (in curly bracket) for the coal-mining data

\begin{tabular}{|c|c|c|c|c|}
\hline Distribution & $\alpha$ & $\theta$ & $\lambda$ & $\eta$ \\
\hline \multirow{2}{*}{ APBXIIP } & $12.25(10.40)$ & $-10.60(2.85)$ & $0.65(0.13)$ & $1.19(0.27)$ \\
& $\{-8.13,32.63\}$ & $\{-13.45,-7.75\}$ & $\{0.40,0.91\}$ & $\{0.66,1.72\}$ \\
\hline \multirow{2}{*}{ PPBXII } & $13.92(3.70)$ & $-(-)$ & $3.07(3.36)$ & $0.12(0.13)$ \\
& $\{6.67,21.17\}$ & $\{-\}$ & $\{-3.52,9.66\}$ & $\{-0.14,0.38\}$ \\
\hline PBXII & $-(-)$ & $-21.99(6.73)$ & $0.58(0.17)$ & $1.25(0.42)$ \\
& $\{-\}$ & $\{-35.18,-8.80\}$ & $\{0.25,0.91\}$ & $\{0.43,2.07\}$ \\
\hline \multirow{2}{*}{ BXII } & $-(-)$ & $-(-)$ & $4.39(1.48)$ & $0.5(0.07)$ \\
& $\{-\}$ & $\{-\}$ & $\{1.49,7.29\}$ & $\{0.36,0.64\}$ \\
\hline
\end{tabular}


Table 8. The AIC, BIC, CAIC, $A^{*}$, and $W^{*}$ statistic for coal-mining data

\begin{tabular}{|c|c|c|c|c|c|c|c|}
\hline Distribution & $-2 l$ & AIC & BIC & CAIC & HQIC & $A^{*}$ & $W^{*}$ \\
\hline APBXIIP & 1432.242 & 1440.242 & 1451.007 & 1440.626 & 1444.608 & 2.6117 & 0.4583 \\
\hline APBXII & 1526.755 & 1532.755 & 1540.829 & 1532.984 & 1536.030 & 2.8623 & 0.4925 \\
\hline PBXII & 1438.772 & 1444.772 & 1452.846 & 1445.0 & 1448.046 & 2.9399 & 0.5136 \\
\hline BXII & 1598.631 & 1602.631 & 1608.013 & 1602.744 & 1604.813 & 3.9302 & 0.6655 \\
\hline
\end{tabular}

Table 9. MLEs and SEs (in Parenthesis), confidence interval (in curly bracket) for the cancer data

\begin{tabular}{|c|c|c|c|c|}
\hline Distribution & $\alpha$ & $\theta$ & $\lambda$ & $\eta$ \\
\hline \multirow{2}{*}{ APBXIIP } & $10.53(9.14)$ & $-4.51(1.39)$ & $1.09(0.32)$ & $1.36(0.46)$ \\
& $\{-7.29,28.35\}$ & $\{-7.23,-1.86\}$ & $\{0.46,1.72\}$ & $\{0.45,2.26\}$ \\
\hline \multirow{2}{*}{ APBXII } & $17.31(5.70)$ & $-(-)$ & $1.85(0.31)$ & $0.52(0.10)$ \\
& $\{6.14,28.48\}$ & $\{-\}$ & $\{1.24,2.46\}$ & $\{0.32,0.72\}$ \\
\hline PBXII & $-(-)$ & $1.13(1.77)$ & $-7.04(0.29)$ & $1.12(0.34)$ \\
& $\{-\}$ & $\{-2.34,4.60\}$ & $\{-7.61,-6.47\}$ & $\{0.45,1.79\}$ \\
\hline BXII & $-(-)$ & $-(-)$ & $0.24(0.36)$ & $2.33(0.04)$ \\
& $\{-\}$ & $\{-\}$ & $\{-0.47,0.95\}$ & $\{2.25,2.41\}$ \\
\hline
\end{tabular}

Table 10. The AIC, BIC, CAIC, $A^{*}$, and $W^{*}$ statistics for cancer data

\begin{tabular}{|c|c|c|c|c|c|c|c|}
\hline Distribution & $-2 l$ & AIC & BIC & CAIC & HQIC & $A^{*}$ & $W^{*}$ \\
\hline APBXIIP & 826.422 & 834.4218 & 845.829 & 834.747 & 839.057 & 0.6264 & 0.0944 \\
\hline APBXII & 851.982 & 857.981 & 866.538 & 858.175 & 861.458 & 1.8386 & 0.2865 \\
\hline PBXII & 834.792 & 840.792 & 849.348 & 840.985 & 844.268 & 1.2370 & 0.1882 \\
\hline BXII & 907.034 & 911.035 & 916.740 & 911.132 & 913.353 & 4.5426 & 0.7475 \\
\hline
\end{tabular}



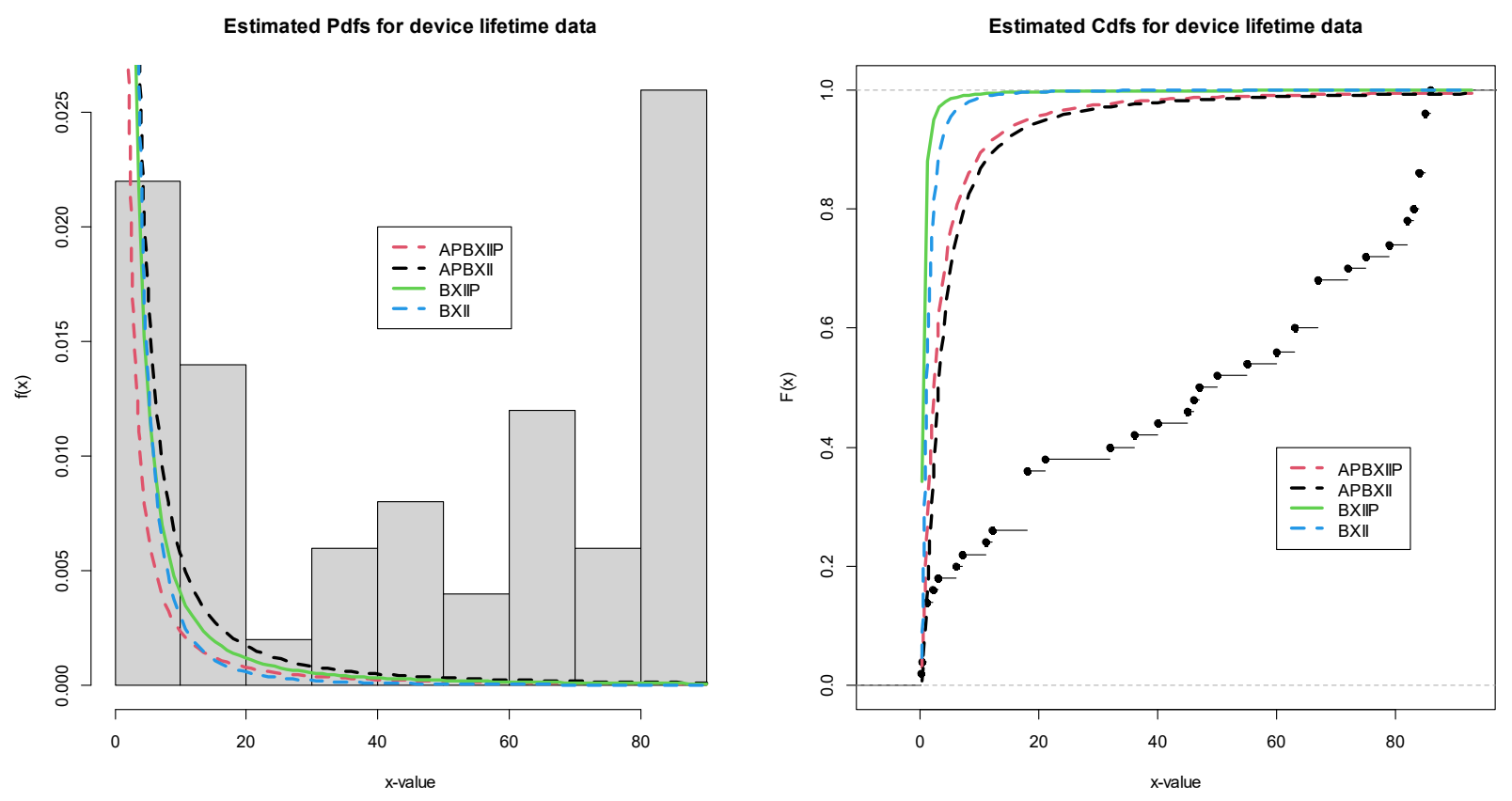

Figure 6. Estimated PDF and CDF function and other competing models for device lifetime data
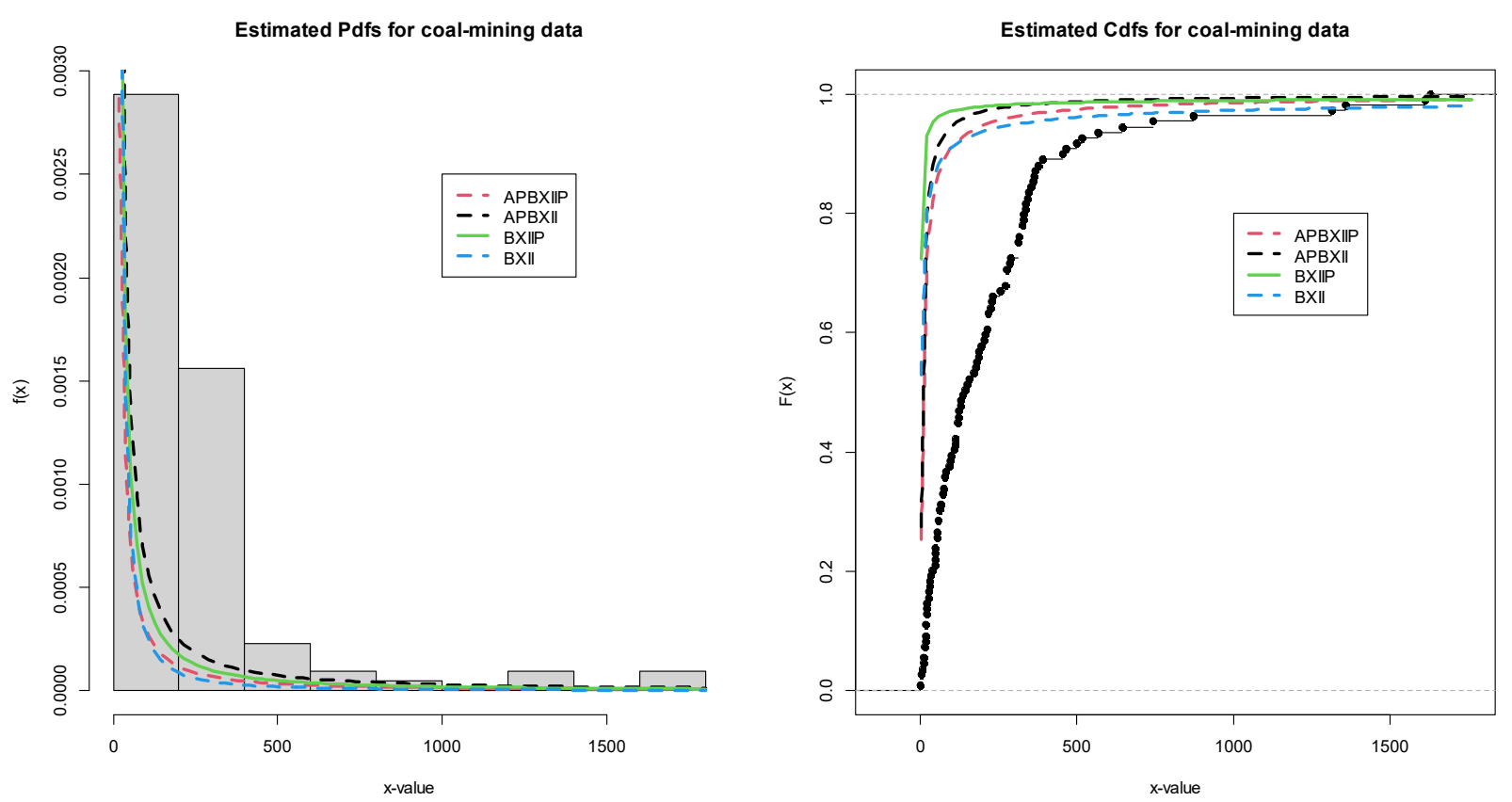

Figure 7. Estimated PDF and CDF function and other competing models for coal-mining data 

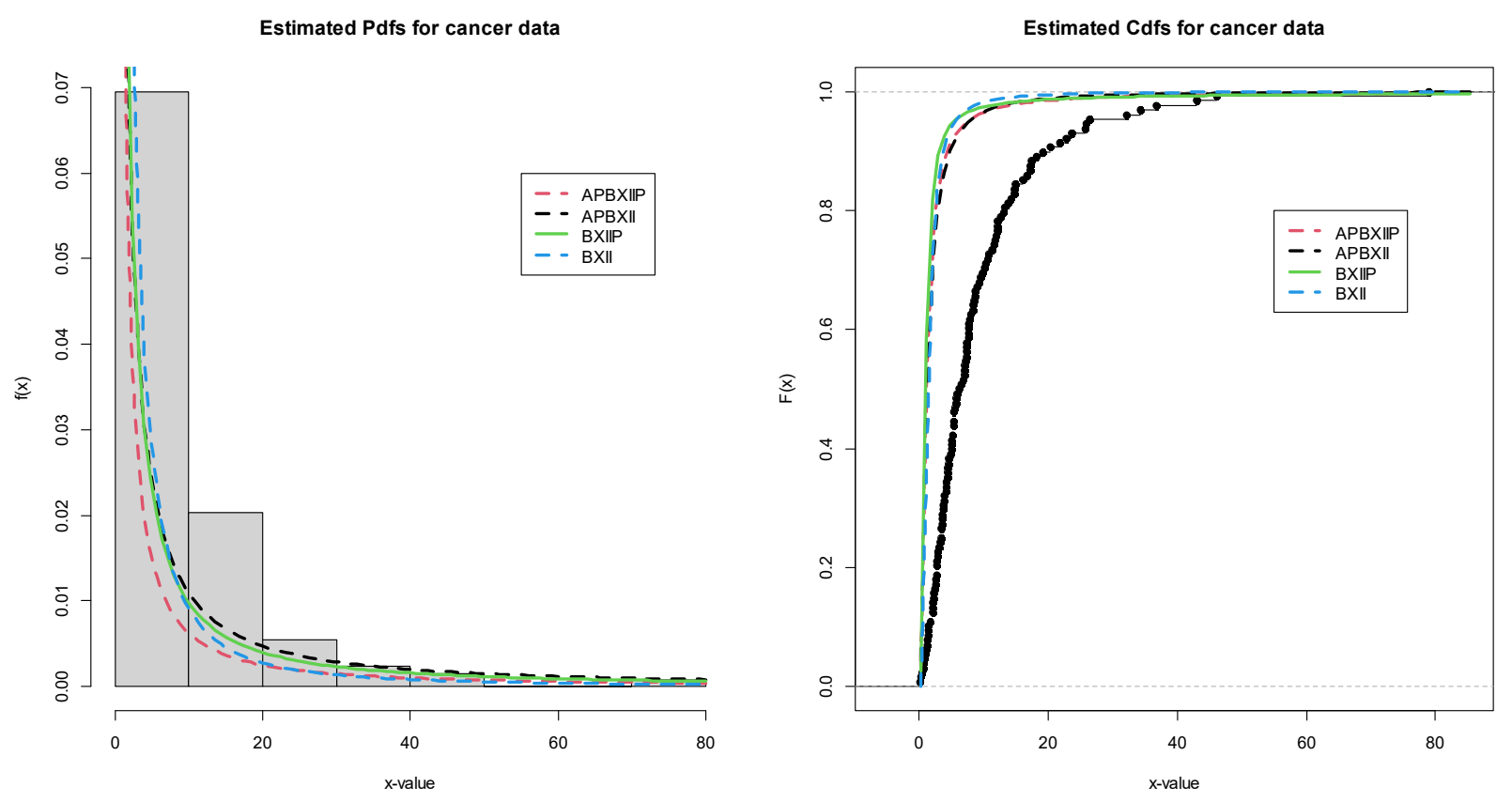

Figure 8. Estimated PDF and CDF function and other competing models for Yarn specimen data

Based on Tables 6, 8 and 10, it is evident that APBXIIP model provides the best fit and can therefore be taken as the best model based on the data considered. Figures 6,7 , and 8 provide more information on the flexibility of the APBXIIP model.

\section{Conclusion}

A new $A P P G$ family of distribution has been introduced to incorporate skewness to a classical distribution functions. We have used that method to the Bur XII distribution functions, and a new four-parameter APBXIIP distribution has been introduced and studied. The proposed distribution has several desirable properties, which enable it use for modeling data that exhibits different shape of the hazard function. Maximum likelihood estimation procedure is used to estimate the values of the unknown parameters. Three data analysis has been performed based on four-parameter $A P B X I I P$ distribution. It is observed that the four-parameter $A P B X I I P$ distribution provides a good fit to the data sets. Monte Carlo simulation is carried out to validate the use of maximum likelihood estimation.

\section{References}

Aarset, M. V. (1987). How to identify bath tub hazard rate. IEEE Trans. Reliab., 36, 106-108. https://doi.org/10.1109/TR.1987.5222310

Afify, A. Z., \& Cordeiro, G. M., Yousof, H. M., Alzaatreh, A., \& Nofal, Z. M. (2016b). The Kumaraswamy transmuted-G family of distributions: properties and applications. Journal of Data Science, 14, 245-270. https://doi.org/10.6339/JDS.201604_14(2).0004

Afify, A. Z., Alizadeh, M., Yousof, H. M., Aryal, G., \&Ahmad, M. (2016a). The transmuted geometric-G family of distributions: theory and applications. Pakistan Journal of Statistics, 32(2), 139-160.

Alizadeh, M., Ghosh, I., Yousof, H. M., Rasekhi, M., \& Hamedani G. G. (2017). The generalized odd generalized exponential family of distributions: properties, characterizations, and applications. Journal of Data Science, 15, 443466. https://doi.org/10.6339/JDS.201707_15(3).0005

Alizadeh, M., Rasekhi, M., Yousof, H. M., \& Hamedani, G. G. (2018). The transmuted Weibull G family of distributions. Hacettepe Journal of Mathematics and Statistics, 47, 1-20. https://doi.org/10.15672/HJMS.2017.440

Altun, E., Yousof, H. M., Chakraborty, S., \& Handique, L. (2018). Zografos-Balakrishnan Burr XII distribution: regression modeling and applications. International Journal of Mathematics and Statistics, 19, 46-70. https://doi.org/10.15672/HJMS.2017.410

Aryal, G. R., \& Yousof, H. M. (2017). The exponentiated generalized-G Poisson family of distributions. Economic Quality Control, 32, 1-17. https://doi.org/10.1515/eqc-2017-0004 
Bourguignon, M., Silva, R.B., \& Cordeiro, G. M. (2014). The Weibull- G family of probability distributions. Journal of Data Science, 12, 53-68. https://doi.org/10.6339/JDS.201401_12(1).0004

Burr, I. W. (1942). Cumulative frequency functions. Annals of Mathematical Statistics, 13, $215-232$. https://doi.org/10.1214/aoms/1177731607

Cordeiro, G. M., \& de Castro, M. (2011). A new family of generalized distributions. Journal of Statistical Computation and Simulation, 81, 883-898. https://doi.org/10.1080/00949650903530745

Cordeiro, G. M., Afify, A. Z., Yousof, H. M., Pescim, R. R., \& Aryal, G. R. (2017). The exponentiated Weibull family of distributions: theory and applications. Mediterranean Journal of Mathematics, 14, 1-22. https://doi.org/10.1007/s00009-017-0955-1

Cordeiro, G. M., Alizadeh, M., \& Marinho, E. P. R. D. (2015). The type I halflogistic family of distributions. Journal of Statistical Computation and Simulation, 86, 707-728. https://doi.org/10.1080/00949655.2015.1031233

Cordeiro, G. M., Alizadeh, M., \& Ortega, E. M. M. (2014). The exponentiated half-logistic family of distributions: Properties and applications. Journal of Probability and Statistics, Article ID 864396, 21 pages. https://doi.org/10.1155/2014/864396

Cordeiro, G. M., Ortega, E. M. M., \& da Cunha, D. C. C. (2013). The exponentiated generalized class of distributions. Journal of Data Science, 11, 1- 27. https://doi.org/10.6339/JDS.2013.11(1).1086

Eugene, N., Lee, C., \& Famoye, F. (2002). The beta-normal distribution and its applications. Communications in Statistics \& Theory and Methods, 31, 497-512. https://doi.org/10.1081/STA-120003130

Gupta, R. C., Gupta, P. L., \& Gupta, R. D. (1998). Modeling failure time data by Lehmann alternatives. Comm. Statist. Theory Methods, 27, 887-904. https://doi.org/10.1080/03610929808832134

Gupta, R. D., \& Kundu, D. (1999). Generalized exponential distributions. Australian and New Zealand Journal of Statistics, 41, 173-188. https://doi.org/10.1111/1467-842X.00072

Hassan, A. S., \& Elgarhy, M. (2016). Kumaraswamy Weibull-generated family of distributions with applications. Advances and Applications in Statistics, 48, 205-239. https://doi.org/10.17654/AS048030205

Kenney, J. F., \& Keeping, E. S. (1962). Mathematics of Statistics. $3^{\text {rd }}$ edition, Part 1, New Jersey.

Korkmaz, M. C., Yousof, H. M., Hamedani G. G., \& Ali, M. M. (2018). The Marshall-Olkin generalized-G Poisson family of distributions, Pakistan Journal of Statistics, 34(3), 251-267.

Lee, E. T., \& Wang, J. (2003). Statistical methods for survival data analysis. John Wiley and Sons. https://doi.org/10.1002/0471458546

Mahdavi, A., \& Kundu, D. A. (2017). A new method for generating distributions with an application to the exponential distribution. Communications in Statistics-Theory and Methods, 46(13), 6543-57. https://doi.org/10.1080/03610926.2015.1130839

Marshall, A. W., \& Olkin, I. (1997). A new method for adding a parameter to a family of distributions with application to the exponential and Weibull families. Biometrika, 84(3), 641-652. https://doi.org/10.1093/biomet/84.3.641

Moors, J. J. (1988). A quantile alternative for kurtosis. J. Royal Statist. Soc. D., 37, $25-32$. https://doi.org/10.2307/2348376

Mudholkar, G. S., \& Srivastava, D. K. (1993). Exponentiated Weibull family for analysing bathtub failure rate data, IEEE Trans. Reliability, 42, 299-302. https://doi.org/10.1109/24.229504

Mudholkar, G. S., Srivastava, D. K., \& Freimer, M. (1995). The exponentiated Weibull family: A reanalysis of the bus-motor-failure data. Technometrics, 37, 436-445. https://doi.org/10.1080/00401706.1995.10484376

Nadarajah, S. (2005). The exponentiated Gumbel distribution with climate application, Environmetrics, 17, $13-23$. https://doi.org/10.1002/env.739

Nadarajah, S., \& Kotz, S. (2006). The exponentiated-type distributions. Acta Appl. Math., 92, 97-111. https://doi.org/10.1007/s10440-006-9055-0

Nadarajah, S.,and Gupta, A. K. (2007). The exponentiated gamma distribution with application to drought data. Calcutta Statistica. Association. Bull., 59, 29-54. https://doi.org/10.1177/0008068320070103

Oluyede, B. O., Pu, S., Makubate, B., \& Qiu, Y. (2018). The Gamma Weibull-G Family of Distributions, To appear in Austrian Journal of Statistics. https://doi.org/10.17713/ajs.v47i1.155

Pescim, R. R., Cordeiro, G. M., Demetrio, C. G. B., Ortega, E. M. M., \& Nadarajah, S. (2012). The new class of 
Kummer beta generalized distributions. Statistics and Operations Research Transactions, 36, 153-180.

Ramos, M. W. A., Marinho, P. R. D., Cordeiro, G., da Silva, R. V., \& Hamedani, G. G. (2015). The Kumaraswamy-G Poisson family of distributions, Journal of Statistical Theory and Applications, 14, 222-239. https://doi.org/10.2991/jsta.2015.14.3.1

Renyi A. (1961). On measures of entropy and information. In: Proceedings of the 4th Fourth Berkeley Symposium on Mathematical Statistics and Probability, 547-561. University of California Press, Berkeley.

Ristic, M. M., \& Balakrishnan, N. (2011). The gamma-exponentiated exponential distribution. Journal of Statistical Computation and Simulation, 82, 1191-1206. https://doi.org/10.1080/00949655.2011.574633

Shannon, C. E. (1951). Prediction and entropy of printed english. Bell system technical journal, 30(1), 50-64. https://doi.org/10.1002/j.1538-7305.1951.tb01366.x

Shirke, D. T., \& C. S., Kakade, C. S. (2006). On exponentiated lognormal distribution. International Journal of Agricultural Statistical Science, 2, 319-326.

Tadikamalla, P. R. (1980). A look at the Burr and related distributions. International Statistical Review, 48, $337-344$. https://doi.org/10.2307/1402945

Yousof, H. M., Afify, A. Z., Alizadeh, M., Butt, N. S., Hamedani, G. G., \& Ali, M. M. (2015). The transmuted exponentiated generalized-G family of distributions. Pakistan Journal Statistics and Operation Research, 11, 441-464. https://doi.org/10.18187/pjsor.v11i4.1164

Zimmer, W. J., Keats, J. B., \& Wang, F. K. (1998). The Burr XII distribution in reliability analysis. Journal of Quality Technology., 30, 386-94. https://doi.org/10.1080/00224065.1998.11979874

Zografos, K., \& Balakrishnan, N. (2009). On families of beta-and generalized gamma-generated distributions and associated inference. Statistical Methodology, 6, 344- 362. https://doi.org/10.1016/j.stamet.2008.12.003

\section{Copyrights}

Copyright for this article is retained by the author(s), with first publication rights granted to the journal.

This is an open-access article distributed under the terms and conditions of the Creative Commons Attribution license (http://creativecommons.org/licenses/by/4.0/). 